\title{
Patrones de diversidad y composición florística de parcelas de evaluación permanente en la selva central de Perú
}

\author{
Diversity patterns and floristic composition of permanent evaluative plots \\ in the Peruvian central forest
}

\author{
José Luis Marcelo-Peña ${ }^{1,2}$ \& Carlos Reynel Rodriguez ${ }^{1}$
}

\begin{abstract}
Resumen
En una muestra de bosque de una hectárea ubicada en la región Junín, provincia de Satipo, a una altura 990 y 1050 $\mathrm{m}$. Se registraron 775 árboles $\geq 10 \mathrm{~cm}$ de DAP, representados en 102 especies, 67 géneros y 37 familias. Desde el punto de vista de la diversidad la zona de estudio posee una riqueza de especies moderada, en contraste a otras muestras estudiadas en selva central de Perú. El presente trabajo también incluye un análisis de siete parcelas de una hectárea ubicadas a lo largo de la gradiente altitudinal en selva central, establecidas en investigaciones previas. De las parcelas instaladas entre 900 y $1500 \mathrm{~m}$ en el piso premontano, el ensamblaje de especies muestra afinidades florísticas entre ellas y con los bosques de Amazonía baja. No obstante, por encima de los $2000 \mathrm{~m}$, la mayor presencia de familias y géneros altoandinos revela marcadas diferencias con el bosque premontano. Las familias Lauraceae, Moraceae, Rubiaceae, Melastomataceae, Urticaceae y Leguminosae, así como, los géneros Ocotea, Ficus, Nectandra, Inga y Miconia son los más ricos en especies en los bosques de selva central del Perú.
\end{abstract} Palabras-claves: diversidad, florística, parcelas, Junín, Perú.

\begin{abstract}
A forest survey in Satipo Province, Region of Junin, from 990 to 1050 m.a.s.l. revealed the presence of 775 trees $\geq 10 \mathrm{~cm}$ dbh belonging to 102 species, 67 genera and 37 families. The study showed the most diverse families as being Moraceae, Lauraceae and Leguminosae, and the most diverse genera as Ficus, Ocotea, Miconia and Inga. A comparative analysis of 1 hectare plots located in premontane forests from 900 to 1150 m.a.s.l. was carried out. In the premontane altitude zone, the species ensemble shows floristic affinities between them and with lowland Amazon forests. However, over 2000 m.a.s.l., there are marked differences on account of greater numbers of high Andean families and genera. Lauraceae, Moraceae, Rubiaceae, Melastomataceae, Urticaceae and Leguminosae, as well as the genera Ocotea, Ficus, Nectandra, Inga and Miconia show high species richness in the montane and premontane forests of Central Peru.
\end{abstract}

Key words: diversity, floristics, plots, Junín, Peru.

\section{Introducción}

Los bosques montanos y premontanos se encuentran entre los 1000-3500 m de altura, en América Latina estos ecosistemas representan aproximadamente 24 millones de ha con porciones importantes en México, Guatemala, Nicaragua, Honduras, en Mesoamérica; y Perú, Colombia, Venezuela, y Argentina en Sudamérica (Kapos et al. 2000). En el Perú existen 15.736 .030 ha (20\% de la superficie total de los bosques del Perú)
(MINAM 2011) y aproximadamente 2.355 .500 ha de superficie es protegida.

Los estudios de bosques tropicales en áreas montañosas han enfatizado que son áreas mundialmente importantes por la riqueza de especies y endemismos (Cleff et al. 1984; Gentry 1989; Rangel 1991; Gentry 1992; Hamilton et al. 1994; Churchill et al. 1995; Bussman 2001). Los bosques montanos confluyen entre las tierras bajas de la Amazonía, y organismos de ambos tipos de bosque

Este artículo tiene material adicional en la versión electrónica.

\footnotetext{
Herbario MOL Forestales, Facultad de Ciencias Forestales, Universidad Nacional Agraria La Molina.

${ }^{2}$ Autor para correspondência: jlmarcelop@lamolina.edu.pe
} 
coexisten en un supuesto bosque premontano (Gentry 1995). Estas áreas forman unidades fitogeográficas potencialmente ricas en especies endémicas y podrían representar las únicas áreas donde el bosque ha persistido durante milenios de años a través de los duros períodos de cambios climáticos (Prance 1973; Brown 1977; Fjeldsa et al. 1999).

La selva central de Perú, tiene un gradiente altitudinal que va desde los $3800 \mathrm{~m}$ hasta los 300 m (Vásquez et al. 2005). En la cima de los andes se encuentran pajonales, y en los pisos inferiores tenemos bosques montanos, premontanos, estacionalmente secos y bosques de amazonía baja. Esfuerzos por conocer la flora arbórea en la zona son escasos. En la década de los 80 por la presencia de fuertes conflictos sociales, la zona perdió interés de parte de investigadores por explorar estas áreas. A partir del 2002 el equipo del Herbario MOL realiza estudios de la vegetación leñosa de los bosques del valle de Chanchamayo (Junín) y el personal de Jardín Botánico del Missouri realiza estudios de la flora en tres áreas naturales protegidas y sus zonas de amortiguamiento en los departamentos de Junín y Pasco (Parque Nacional Yanachaya Chemillén, el bosques de protección San Matías-San Carlos y la Reserva comunal Yanesha) (Antón \& Reynel 2004; Vásquez et al. 2005).

En el Perú, estudios de evaluación permanente de los árboles en parcelas de una hectárea se iniciaron a finales de los 80 e inicios de los noventa. Las cifras más altas de riqueza y diversidad del mundo han sido registradas en los bosques amazónicos. Por ejemplo, en áreas cercanas a Iquitos (al noroeste del Perú) se registraron entre 280 a 300 especies de árboles con diámetro a la altura del pecho (DAP) $\geq 10 \mathrm{~cm}$. Así también, en Yasuní (Ecuador) se registraron 253 a 283 especies (Gentry 1988a,b; Phillips et al. 1994; Ter Steege at al. 2000; Pitman et al. 2002; Salgado et al. 2004; Valencia et al. 2004). La zona sur de la Amazonia del Perú (Madre de Dios, Cusco), es conocida por presentar una riqueza y diversidad de árboles leñosos entre moderada a baja (Gentry 1988a,b; Phillips et al. 1994). No obstante, estudios recientes muestran inusuales valores de diversidad cercanos a los registrados en Loreto (Huamantupa 2010).

Estudios de los bosques de Selva Central, empleando parcelas permanentes de una hectárea, fueron instaladas en la gradiente altitudinal a 1050 , $1150,1500,2100$ y $2275 \mathrm{~m}$, y se reportan valores de diversidad arbórea que oscilan entre 90 y 147 especies con DAP $\geq 10$ cm (Antón \& Reynel 2004; Reynel \& Honorio 2004; Reynel \& Antón 2004; Caro et al. 2004).

En la zona montana y premontana de selva central, la principal actividad es la agrícola, se cultiva café, cacao yuca, plátano, maíz, arroz, frijol, cítricos, piñas y otros frutales. Por estas actividades gran parte de la superficie de bosque ha sido deforestada. Así también, la actividad forestal se constituyó en uno de los rubros económicos de mayor importancia en el pasado. Se inició hacia 1920, consumiendo las maderas más valiosas de los ricos bosques existentes (Antón \& Reynel, 2004), dentro de ellas Cedrela odorata L. "cedro" y árboles de "ulcumano" o "romerillo" del género Retrophyllum y Podocarpus. Hacia los años 80 Chanchamayo. Todavía seguía constituyéndose un área importante de producción maderera, aportando el $10 \%$ de la producción de madera aserrada del país (Casas 1989). Actualmente la tala selectiva se realiza para aprovechar Cedrelinga catenaeformis (Ducke) Ducke "tornillo", Cedrela fissilis Vell. "cedro", Virola spp. "cumala", Aspidosperma macrocarpon Mart. "pumaquiro", Juglans neotropica Niels "nogal", Guarea guidonia (L.) Sleumer "requia", Terminalia amazonia (J.F. Gmel) Exell "yacushapana", Nectandra spp. y Ocotea spp. "moena". Por ahora el panorama de destrucción sigue vigente. No obstante, el conocimiento de la biodiversidad y de los aspectos relacionados a la ecología y el manejo de los recursos del bosque, es aún precario.

En este contexto, la investigación se propuso los siguientes objetivos (i) conocer la composición florística y la diversidad arbórea de un relicto de bosque del distrito de Río Negro, Satipo, Junín, y (ii) analizar las afinidades florística de la zona de estudio con otras muestras de bosque de selva central. Se espera que los resultados sirvan de base para continuar investigaciones sobre la dinámica, la ecología y la silvicultura de la flora arbórea de esta parte del país.

\section{Materiales y Métodos}

Área de estudio

La zona de estudio se encuentra políticamente ubicada al este del departamento de Junín, provincia de Satipo, distrito de Río Negro, en el Fundo Santa Teresa propiedad de la Universidad Nacional Agraria La Molina. La parcela se instaló a una altitud que va desde 990 a $1050 \mathrm{~m}$, entre las coordenadas $11^{\circ} 10^{\prime} 0.9^{\prime}$ 'S y $74^{\circ} 38^{\prime} 55^{\prime \prime} \mathrm{W}$ (Fig. 1). 
Siguiendo el criterio de Brack \& Mendiola (2000), la parcela de Santa Teresa (P-ST) se encuentra en el piso inferior de la Ecorregión de la Selva Alta de 800 a 1300 m. Siguiendo el criterio de Holdridge (1978), la zona de estudio se encuentra dentro de la zona de vida bosque húmedo Premontano Tropical (bh-PM). El clima del área se caracteriza por tener precipitaciones anuales medias alrededor de $1.757 \mathrm{~mm}$. La temperatura media anual es de $21.5^{\circ} \mathrm{C}$. Las temperaturas mínimas se registran en los meses de Enero y Febrero y las más altas en los meses de Agosto y Septiembre, definiendo un clima cálido y húmedo de selva alta (ONERN 1976). La topografía de la zona varía de ondulada a empinada donde predominan laderas y colinas con pendientes moderadas. Los suelos son de profundidad media a profunda y de texturas franco a pesada. $\mathrm{El} \mathrm{pH}$ del suelo oscila de ácido a neutro y pertenecen a las órdenes de los Entisoles, Inceptisoles y Alfisoles con fertilidad natural de baja a media (ONERN 1976).

Otras parcelas incluidas en este manuscrito se encuentran también en la zona de vida bosque húmedo premontano tropical: (1) parcela La Génova ladera (P-GL) (2) parcela La Génova cima (P-GC), (3) parcela microcuenca El Tirol de ladera (P-MTL) y (4) parcela Los Cedros de Pampa Hermosa (P-CPH), estas fueron ubicadas en el distrito de San Ramón y La Merced, provincia de Chanchamayo, departamento de Junín, en alturas que oscilan entre los 1075 y $1500 \mathrm{~m}$. Solo las parcelas del sector Pichita se encuentran sobre los $2000 \mathrm{msnm}$ (parcela Pichita ladera (P-PL) y parcela Pichita ribera (P-PR) (San Ramón)), y ocupan la zona de vida bosque muy húmedo montano bajo tropical. El clima de esta zona esta escasamente documentada y sus valores son de extrapolaciones (ONERN 1976; INRENA 1995) y tienen relación por la inaccesibilidad de estos ambientes. Con las observaciones mencionadas los parámetros básicos de temperatura y precipitación, está dentro de los siguientes valores, la temperatura promedio anual oscila entre $15-19^{\circ} \mathrm{C}$ y la precipitación total anual promedio oscila entre $1500-3000 \mathrm{~mm}$.

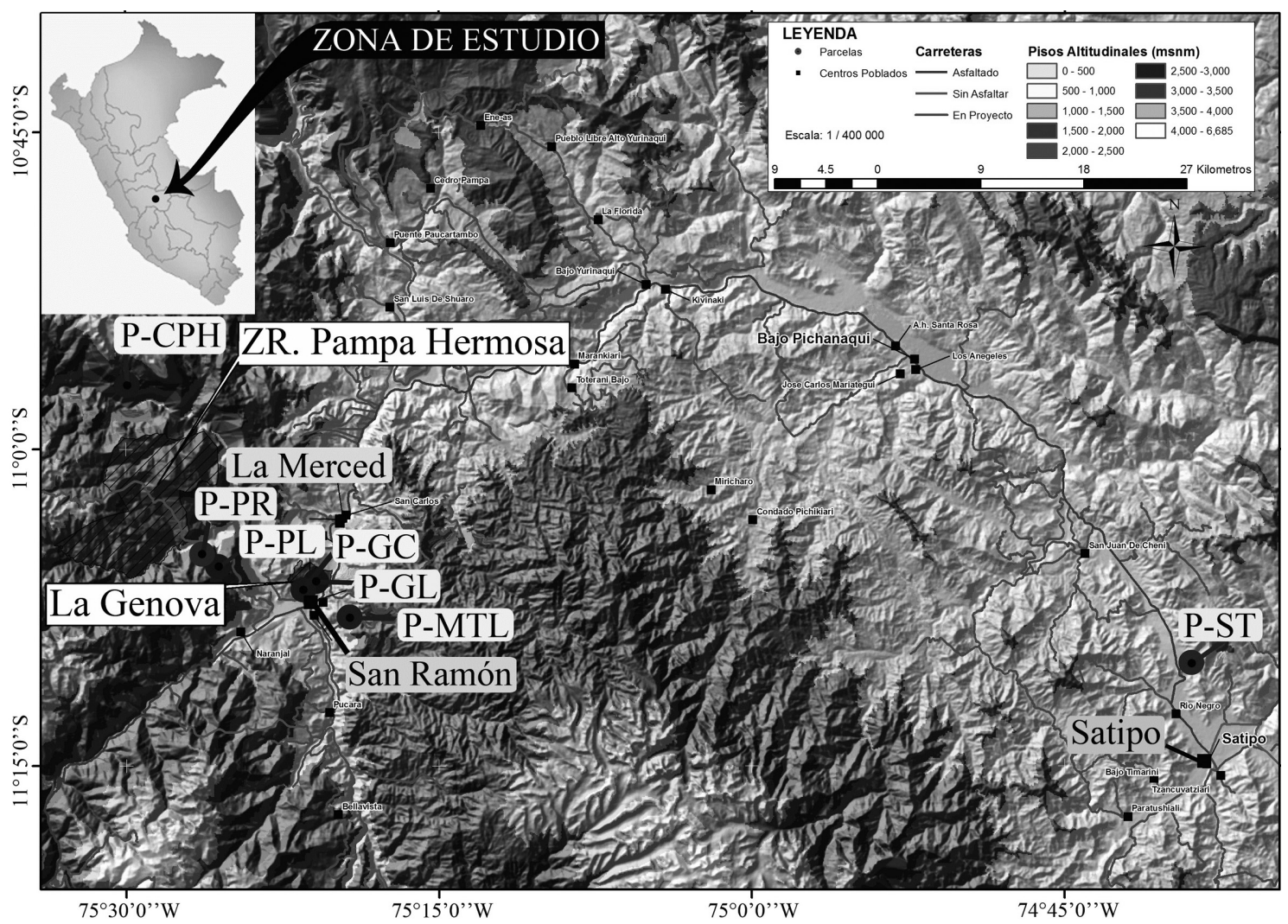

Figura 1 - Mapa de ubicación de las parcelas instaladas en selva central tratadas en el estudio.

Figure 1 - Map showing the location of the 0.1 ha forest plots included in the present study. 


\section{Metodologia}

Para la elección del área de estudio se estratificó el bosques, luego hicimos recorridos por las diferentes formaciones y se seleccionó un área de colina que posee pendientes que van desde $20^{\circ}-50^{\circ}$. Esta área fue elegida por poseer mejor estado de conservación y encontrarse relativamente alejada de caminos o trochas. Para el establecimiento, delimitación, plaqueado y registro de los árboles, se siguió la metodología propuesta por Phillips \& Baker (2006).

La localización, delimitación, plaqueado y registro de los árboles se realizó entre Febrero del 2007 hasta enero de 2008. Las colecciones botánicas se realizaron desde abril de 2007 hasta enero del 2009. En la primera etapa del trabajo solo se realizó la colección de los árboles menores de 10 $\mathrm{m}$ de alto. Para la colección de los árboles de más de $10 \mathrm{~m}$ de alto, se utilizaron los subidores "pata de loro", cinturón de seguridad y tijera telescópica. A medida que nos familiarizamos con las especies, las más abundantes ya no se colectaron. Esta tarea se realizó solo en taxones que conducen a errores de identificación como Miconia, Inga, Virola o Lauráceas. Además, de todos aquellos que no pudieron ser identificados en campo.

El proceso de herborización siguió la metodología propuesta por Bridson \& Forman, (1999). Todas las muestras botánicas fueron depositadas en el Herbario MOL. La taxonomía de familias y géneros está basada en el APG II (2003)

\section{Análisis de datos}

Se confeccionó una base de datos de todos los individuos registrados en el programa EXCEL (Microsoft 2000). Con este programa se prepararon los gráficos de estructura y los cálculos de estructura del bosque. También se utilizó el programa PRIMER v5 (Clarke \& Gorley 2001), para calcular el índice de diversidad de Fisher. Adicionalmente, se calculó el índice de valor de importancia por familia (IVIF) y el índice de valor de importancia por especie (IVI), de acuerdo a la fórmula de Mori et al. (1983) y Curtis \& McIntosh (1951).

Análisis comparativo de áreas de bosque en selva central (San Ramón, La Merced y Satipo)

Con los registros de la zona de estudio se construyó una matriz con datos de abundancia, y se adicionaron seis inventarios de otras parcelas
(Apéndice en versión on line) de una hectárea (Antón \& Reynel 2004; Reynel \& Honorio 2004; Reynel \& Antón, 2004; Caro et al. 2004; La Torre 2003). Para el análisis se revisaron los duplicados de herbario de la familia Lauraceae registrados en las parcelas Pichita ladera (P-PL) y Cedros de Pampa Hermosa (P-CPH). La revisión de esta familia se hizo por el alto número de morfoespecies, que usualmente sobre valora el número de especies en los inventarios. En total se revisaron 164 duplicados. Todas las especies de Lauraceae registradas en las parcelas fueron fotografiadas y están disponibles en el Herbario MOL. También del análisis se excluyeron los taxa desconocidos a nivel de familia. La matriz final incluyó siete parcelas y 496 especies, 214 géneros y 76 familias.

Se aplicó análisis multivariados a la matriz para explorar las afinidades florísticas entre las parcelas. Utilizamos los datos de abundancia que fueron transformados logarítmicamente $[\log (x+1)]$, para compensar el efecto de las especies dominantes y menos numerosas o raras en los resultados estadísticos. La matriz transformada fue sometida a una ordenación de Escalamiento multidimensional (MDS), usando el índice de similaridad BrayCurtis. Este método de similaridad traduce medidas de semejanza de sitio por sitio, en una matriz interior de distancia euclideana de distancia entre sitios en una ordenación, con estrés (un coeficiente no-paramétrico de regresión), la medida de buen estado está entre las dos medidas cortas de reemplazo. También se prepararon matrices de presencia/ausencia y de géneros. Para los análisis multivariados se utilizó el programa PRIMER v5 (Clarke \& Gorley 2001). El gráfico de dispersión de especies fue elaborado con el programa InfoStat versión 2012 (Di Rienzo et al. 2012)

\section{Resultados}

Diversidad y composición florística

Se registraron 102 especies, 67 géneros y 37 familias. Las seis familias más diversas ordenadas en forma decreciente fueron Moraceae (20 especies), Lauraceae y Leguminosae (11), Melastomataceae (7), Euphorbiaceae (5) y Cecropiaceae (4), las demás familias presentan menos de tres especies. Los cuatro géneros más diversos ordenados en forma decreciente son: Ficus (10), Ocotea (8), Miconia (6) y Inga (4).

Para cuantificar la diversidad se tomó como referencia el índice de diversidad de Fisher, este 
índice caracteriza comunidades bióticas que contienen pocas especies que son abundantes y muchas que son escasas, además, valoriza la diversidad independientemente del área y del tamaño de la muestra (Fisher et al.1943). El valor de Fisher para la muestra de estudio es de 31. El coeficiente de mezcla fue de 7.6 (775/102); es decir, en promedio existen cerca de ocho individuos por especie.

\section{Densidad y frecuencia}

El número total de individuos fue de 775 , las seis familias más abundantes ordenadas en forma decreciente son: Annonaceae (121 individuos), Urticaceae (92), Melastomataceae (89), Euphorbiaceae (76), Leguminosae (65) y Vochysaceae (50), estas familias poseen el $73.4 \%$ de total de individuos (Fig. 2). La especie más abundante fue Guatteria hyposericea Diels (119), le siguen en orden decreciente Pouroma minor Benoist (87), Alchornea glandulosa Poeppig (62), Vochysia venulosa Warming. (50), Henrietella sylvestris Gleason (46) y Casearia arborea (Rich) Urban (24). Son especies raras 41 especies con un solo individuo, incluye un solo registro de Socratea exorrhiza (Mart.) H. Wendl. (Arecaceae).

Las especies más abundantes también son las más frecuentes, ordenadas en forma decreciente tenemos a $P$. minor registrada en 24 subparcelas, G. hyposericea registrada en 22 subparcelas, A. glandulosa registrada en 20 subparcelas, $H$. sylvestris registrada en 18 subparcelas, $V$. venulosa registrada en 17 subparcelas y $T$. lawrancei registrada en 16 subparcelas.

Distribución de diámetro y área basal

El diámetro máximo alcanzado en la zona de estudio fue de $59.5 \mathrm{~cm}$. Las clases diamétricas forman la clásica “j”" invertida (Fig. 3), con una alta representación en los diámetros menores que va decreciendo exponencialmente hacia los de mayor tamaño. La clase diamétrica $10-20 \mathrm{~cm}$, concentra el mayor número de individuos con un total de 570 de 90 especies. En la clase diamétrica $40-50 \mathrm{~cm}$, se registraron tres especies con cuatro individuos, Apeiba membranacea Spruce ex Benth. (2), A. glandulosa y Ficus ypsilophlebia Dugand. Solo A. membranacea y Ficus gomelleira Kunth \& C. D. Bouché, poseen un DAP $>50 \mathrm{~cm}$.

El área basal total del área de estudio es $21.60 \mathrm{~m}^{2}$, las especies con mayor área basal ordenadas en forma descendente son: $G$. hyposericea $\left(2.78 \mathrm{~m}^{2}\right)$, P. minor $\left(2.29 \mathrm{~m}^{2}\right), A$. glandulosa $\left(2.10 \mathrm{~m}^{2}\right), V$. venulosa $\left(1.57 \mathrm{~m}^{2}\right)$, H. sylvestris $\left(1.23 \mathrm{~m}^{2}\right)$, Trattinnickia lawrancei Standl. $\left(0.76 \mathrm{~m}^{2}\right)$ y C. arborea $\left(0.38 \mathrm{~m}^{2}\right)$. Estas especies poseen el $51.60 \%$ del área basal total.

\section{Estratificación vertical}

La estructura vertical está divida en tres estratos, dosel suprimido, dosel medio y dosel superior. El dosel suprimido corresponde a todos los individuos $\leq 9 \mathrm{~m}$ de alto, posee 62 individuos de 34 especies y representa el $8 \%$, las especies más abundantes fueron $A$. glandula y $P$. minor. El dosel medio corresponde a todos los individuos 10-20 m, posee 629 individuos de 89 especies y representa el $81.2 \%$, las especies más abundantes fueron $G$. hyposericea, $P$. minor y $V$. venulosa. El dosel superior posee 84 individuos de 27 especies y representa el $10.8 \%$, las especies má abundantes

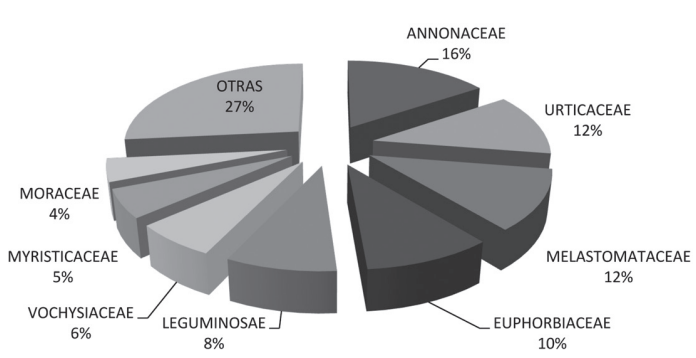

Figura 2 - Familias más abundantes del bosque Santa Teresa, Portillo Alto, Río Negro, Satipo.

Figure 2 - Most abundant families from the forest of Santa Teresa, Portillo Alto, Río Negro, Satipo.

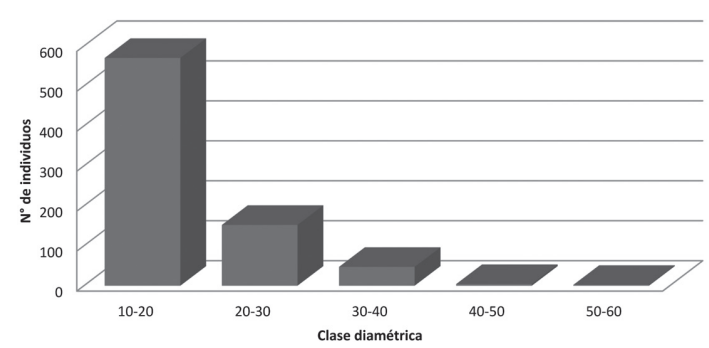

Figura 3 - Curva de distribución de individuos por clases diamétricas de 1.0 ha de bosque de Santa Teresa, Portillo Alto, Río Negro, Satipo, mostrando la tradicional J invertida.

Figure 3 - Distribution curve of individuals vs. diametric classes in a 1.0 ha forest plot in Santa Teresa, Portillo Alto, Río Negro, Satipo, showing the characteristic inverted J. 
fueron $V$. venulosa, P. minor y G. hyposericea. Índice de valor de importancia (IVI)

Las ocho especies más importantes ordenadas en forma decreciente son $G$. hyposericea (33.75), $P$. minor (27.84), A. glandulosa (22.71), $V$. venulosa (17.99), H. sylvestris (16.16), T. lawrancei (10.75), Jacaranda macrocarpa (A. DC.) Bureau \& Schumann (8.68) y C. arborea (8.36). Estas especies representan el $48.75 \%$ del total de especies registradas en el estudio (Tab. 1). El índice de valor de importancia por familia (IVIF), es el resultado de sumar los valores relativos de la abundancia, dominancia y la diversidad de una familia (Mori et al. 1983). Las cinco familias más importantes ordenadas en forma decreciente son, Melastomataceae (31.71), Annonaceae (30.6), Urticaceae (27.57), Moraceae (27.41),
Euphorbiaceae (26.84), Leguminosae (26.6) y Lauraceae (17.37). Estas familias representan el $83.73 \%$ del total de las familias registradas.

\section{Beta diversidad en Selva Central}

El análisis de similaridad de Bray-Curtis y la clasificación de MDS, reconoció cuatro grupos distintos de especies de árboles (1) la muestra de Santa Teresa (P-ST), al sureste del diagrama de dispersión, (2) las muestras de bosque de Pichita al suroeste (P-PL, P-PR), (3) la muestra de bosque de los Cedros de Pampa Hermosa (P-CPH) al noroeste y (4) las muestras de bosque de La Génova (P-GC, $\mathrm{P}-\mathrm{GL}$ ) con la muestra de bosque de la microcuenca El Tirol (P-MTL) al centro (Fig. 4). Pequeñas diferencias fueron detectadas con la matriz de presencia/ausencia de todos los taxa, separando

Tabla 1 - Índice de valor de importancia de las 20 especies más importantes registradas en una hectárea del bosque de San Teresa, Satipo. AB. ABS. =Abundancia absoluta, AB. REL.=Abundancia relativa, FR. ABS. $=$ Frecuencia absoluta, FR. REL. $=$ Frecuencia relativa, DOM. ABS. $=$ Dominancia absoluta, DOM. REL.=Dominancia relativa, IVI= Índice de valor de importancia.

Table 1 - Importance Value Index of the 20 most important species registered in a 1.0 ha forest plot in Santa Teresa, Satipo. AB. ABS.= Absolute abundance, AB. REL.= Relative abundance, FR. ABS.= Absolute frequency, FR. REL.= Relative frequency, DOM. ABS.= Absolute dominance, DOM. REL. = Relative dominance, IVI = Importance Value Index.

\begin{tabular}{|c|c|c|c|c|c|c|c|c|c|}
\hline $\mathbf{N}^{\circ}$ & ESPECIES & Ab. Ab & Ab. Rel. & F. Ab. & F. Rel. & Do. Ab. & D. Rel. & IVI & IVI\% \\
\hline 1 & Guatteria hyposericea Diels & 119 & 15,35 & 22 & 5,49 & 2,788 & 12,9 & 33,75 & 11,25 \\
\hline 2 & Pourouma minor Benoist & 87 & 11,23 & 24 & 5,99 & 2,296 & 10,63 & 27,84 & 9,28 \\
\hline 3 & Alchornea glandulosa Poepp. & 62 & 8 & 20 & 4,99 & 2,1 & 9,72 & 22,71 & 7,57 \\
\hline 4 & Vochysia venulosa Warm. & 50 & 6,45 & 17 & 4,24 & 1,576 & 7,3 & 17,99 & 6 \\
\hline 5 & Henrietella sylvestris Gleason & 46 & 5,94 & 18 & 4,49 & 1,24 & 5,74 & 16,16 & 5,39 \\
\hline 6 & Trattinnickia lawrencei Standl. & 24 & 3,1 & 15 & 3,74 & 0,739 & 3,42 & 10,26 & 3,42 \\
\hline 7 & Jacaranda glabra (A. DC.) Bureau \& Schumann & 19 & 2,45 & 9 & 2,24 & 0,861 & 3,99 & 8,68 & 2,89 \\
\hline 8 & Casearia arborea (Rich.) Urb. & 24 & 3,1 & 14 & 3,49 & 0,384 & 1,78 & 8,36 & 2,79 \\
\hline 9 & Miconia barbeyana Cogniaux & 18 & 2,32 & 7 & 1,75 & 0,898 & 4,16 & 8,22 & 2,74 \\
\hline 10 & Virola sebifera Aub. & 18 & 2,32 & 13 & 3,24 & 0,465 & 2,15 & 7,72 & 2,57 \\
\hline 11 & Apeiba membranacea Spruce ex Bentham & 10 & 1,29 & 7 & 1,75 & 0,815 & 3,77 & 6,81 & 2,27 \\
\hline 12 & Virola elongata (Benth.) Warburg & 16 & 2,06 & 11 & 2,74 & 0,408 & 1,89 & 6,7 & 2,23 \\
\hline 13 & Cedrelinga cateniformis (Ducke) Ducke & 15 & 1,94 & 9 & 2,24 & 0,494 & 2,29 & 6,47 & 2,16 \\
\hline 14 & Cordia sp. & 14 & 1,81 & 7 & 1,75 & 0,502 & 2,32 & 5,87 & 1,96 \\
\hline 15 & Cassia grandis L. f. aff. & 15 & 1,94 & 8 & 2 & 0,279 & 1,29 & 5,22 & 1,74 \\
\hline 16 & Inga thibaudiana DC. & 10 & 1,29 & 10 & 2,49 & 0,202 & 0,94 & 4,72 & 1,57 \\
\hline 17 & Cabralea canjerana (Vell.) Martius & 11 & 1,42 & 9 & 2,24 & 0,208 & 0,96 & 4,62 & 1,54 \\
\hline 18 & Miconia sp. & 11 & 1,42 & 7 & 1,75 & 0,192 & 0,89 & 4,05 & 1,35 \\
\hline 19 & Byrsonima sp. & 9 & 1,16 & 5 & 1,25 & 0,337 & 1,56 & 3,97 & 1,32 \\
\hline 20 & Capirona decorticans Spruce & 10 & 1,29 & 7 & 1,75 & 0,142 & 0,66 & 3,7 & 1,23 \\
\hline
\end{tabular}


$\mathrm{P}-\mathrm{CPH}$

Stress: 0.01

P-ST

Figura 4 - Diagrama de ordenación del análisis de MDS de siete parcelas de 01 ha en La Merced, San Ramón y Satipo, selva central de Perú.

Figure 4 - MDS analysis ordination diagram in seven 1.0 ha forest plots in La Merced, San Ramon and Satipo.

ligeramente los bosques de colina de la microcuenca el Tirol, de las muestras de bosque de cresta y colina de La Génova. Tomando como referencia la matriz de géneros, también se reconocen las cinco agrupaciones diferentes de árboles.

\section{Discusión}

De la diversidad y

la composición florística

Este estudio proporciona información adicional sobre la composición florística de los bosques premontanos de la región amazónica. Los bosques premontanos de Selva Central incluyen árboles conspicuos encontrados a lo largo de la Amazonía baja, estos son: G. hyposericea, $P$. minor, V. venulosa, $H$. sylvestris, C. arborea, Virola sebifera Aublet., J. glabra y C. cateniformis.

Estudios realizados en Selva Central, empleando la metodología de parcelas permanentes de una hectárea reportan una diversidad que oscila entre 90 y 147 especies con DAP $\geq 10 \mathrm{~cm}$ (Antón \& Reynel 2004; Reynel \& Honorio 2004; Reynel \& Antón 2004; Caro et al. 2004). Las 102 especies registradas en el presente estudio se encuentra por debajo de la diversidad encontrada en Selva central, excepto, por la muestra de bosque de La Génova ladera (P-GL) (Tab. 2), que es menor a la diversidad registrada en este estudio.

¿Que factores han influenciado en los bosques de Santa Teresa, para poseer niveles de diversidad bajos, si se encuentran en el piso premontano donde el traslape de especies amazónicas y especies andinas incrementan los valores de la diversidad? Se asume (a) que la zona de estudio es de naturaleza transicional, por la alta densidad Pourouma y Alchornea, géneros escasos en formaciones primarias pero abundantes y frecuentes en bosques secundarios, (b) la carencia de lianas con diámetros $\geq 10 \mathrm{~cm}$ de DAP de las familias Bignoniceae, Sapindaceae, Leguminosae, Hippocrateaceae, Menispermaceae y Malphigiaceae (Gentry 1985), que incrementan los niveles de diversidad, (c) la presión sobre el bosque (tala selectiva por más de 90 años) por acción antrópica, que ha destruido poblaciones de otras especies. Esta perturbación histórica podría estar potencialmente afectada por gradientes ambientales de humedad que pueden modificar la distribución de los árboles y los patrones de diversidad (Clinebell at al. 1995). Estas características evidencian la baja diversidad del bosque en estudio.

Gentry (1982a), empleando un mayor número de parcelas de una décima de hectárea y esparcidas en el neotrópico, intentó determinar los factores ecológicos que se podrían correlacionar con una diversidad alta de especies de plantas, llegando a la conclusión que la diversidad aumentaba con la cantidad y la equitabilidad de la precipitación, y en menor grado, con la fertilidad del suelo. No obstante, Oliveira \& Mori (1999), al estudiar áreas más secas, estacionales y con suelos pobres, ubicados en las cercanías de Manaos (en el centro de la Amazonía), encontraron valores relativamente altos de riquezas de especies de árboles: de 280-285 especies por hectárea. Berry (2002), afirma que el conocimiento que se tiene sobre la composición florística de la región amazónica sigue siendo extremadamente pobre. A su vez esta deficiencia limita la posibilidad de hacer generalizaciones acerca de los patrones de la diversidad de los bosques amazónicos, ya sea con propósitos científicos o de conservación. Por lo tanto, es clara la necesidad de realizar muestreos a gran escala y de uniformizar metodologías, para poder analizar en forma adecuada, lo patrones de diversidad y endemismos.

Las familias más diversas reportadas en parcelas de una hectárea en los bosques premontanos (1075 y $1150 \mathrm{msnm})$ de selva central, fueron Lauraceae, Moraceae, Leguminosae, Rubiaceae, Cecropiaceae, Euphorbiaceae y Melastomataceae (Reynel \& Antón 2004; Antón \& Reynel 2004; Caro et al. 2004). En este estudio, también Moraceae, Lauraceae, Leguminosae, Melastomataceae y 
Euphorbiaceae, son las familias más diversas. A pesar que los criterios de evaluación fueron diferentes, hay semejanza florística con muestras de bosque de la región neotropical entre los 800 y 1500 m (Gentry, 1985), donde Leguminosae, Moraceae, Lauraceae y Rubiaceae son las más diversas.

Familias más diversas en parcelas bosques montanos (1500-2275 msnm), fueron Lauraceae, Moraceae, Melastomataceae, Melicaceae, Rubiaceae, Myrtaceae, Myristicaceae, Euphorbiaceae, Cyatheaceae y Cunoniaceae (Reynel \& Honorio 2004; Reynel \& Antón 2004; La Torre 2003). Los patrones de composición florística a estas alturas difieren en contraste con los bosques premontanos, por la mayor presencia de familias del piso montano. A la luz de nuestros resultados Lauraceae y Moraceae son las familias más ricas en especies en los bosques de selva central, en la zona se pueden encontrar 13.29 especies en promedio para la primera y 10.5 especies en promedio para la segunda (Tab. 3).

Los géneros más diversos reportados en parcelas de bosques premontanos (1075 y 1150 msnm) en selva central, fueron Ocotea, Inga, Ficus, Cecropia, (Reynel \& Antón 2004; Antón \& Reynel 2004; Caro et al. 2004). Los géneros más diversos de los bosques montanos (1500-2275 msnm) de Chanchamayo, fueron Ficus, Miconia, Nectandra, Ocotea, Aniba, Cecropia, Myrsine y Guarea. En la P-ST también Ficus, Ocotea, Miconia e Inga son los más diversos. Las afinidades florísticas a nivel de género son semejantes para bosques ubicados en el piso premontano. Con los bosques montanos solo hay afinidades florísticas con las familias más diversas. No obstante, la alta frecuencia de géneros altoandinos como Weinmannia, Brunellia, Cyathea, Prunus, Myrcianthes, Calyptrantes, Miconia y Hyeronima, marcan diferencias entre el piso premontano y montano en selva central. En base a nuestro análisis, Ocotea y Ficus son los géneros más diversos en los bosques de selva central, en parcelas de una hectárea se pueden encontrar 6.8 especies en promedio, para la primera y 5.5 especies en promedio para la segunda (Tab. 4).

Gentry (1992) indica que las especies de Melastomataceae, crecen de tamaños considerables en bosques montanos maduros de altas alturas, mientras que en elevaciones más bajas estas especies se quedan a menudo como arbustos hemiepífitos. Miconia es un género usualmente arbustivo, especies arbóreas mayormente se registran en bosques montanos sobre los $2000 \mathrm{~m}$ (Reynel \& Honorio 2004), la P-ST por ubicarse en una zona de traslape de vegetación montana y amazónica evidencia su presencia.

Especies abundantes registradas en tres parcelas de una hectárea en selva central a $1150 \mathrm{~m}$ fueron Inga cinnamomea Benth. (34 individuos), Trophis caucana (Pittier) C.C: Berg y Trema micrantha (L.) Blume (23), Batocarpus costaricensis Standl. \& L.O. Williams (19), Pseudolmedia laevis (Ruiz \& Pav.) J.F. Macbride

Tabla 2 - Riqueza de familia, géneros y especies de bosques pre-montanos y montanos de Selva central (La Merced, San Ramón y Satipo), en parcelas de 01 ha, con árboles $\geq 10 \mathrm{~cm}$ de DAP. Tomado de Antón \& Reynel (2004); Reynel \& Honorio (2004); Reynel \& Antón (2004); Caro et al. (2004) y La Torre (2003). Los números en negrita son los resultados del presente estudio. Table 2 - Richnes at family, genus and species levels in pre-montane and montane forests of Amazonian Central Peru (La Merced, San Ramon and Satipo), in 1.0 ha plots, with trees $\geq 10 \mathrm{~cm}$ de DAP. Taken from Antón \& Reynel (2004); Reynel \& Honorio (2004); Reynel \& Antón (2004); Caro et al. (2004) and La Torre (2003). Numbers shown in bold are the results of the present study.

\begin{tabular}{lccccccc}
\hline & P-ST & P-PL & P-PR & P-GC & P-GL & P-MTL & P-CPH \\
\hline$N^{\circ}$ familias & $\mathbf{3 7}$ & 42 & 39 & 39 & 28 & 40 & 35 \\
$\mathrm{~N}^{\circ}$ Géneros & $\mathbf{6 7}$ & 82 & 83 & 83 & 55 & 90 & 67 \\
$\mathrm{~N}^{\circ}$ especies & $\mathbf{1 0 2}$ & 147 & 120 & 121 & 90 & 124 & 144 \\
$\mathrm{~N}^{\circ}$ de individuos & $\mathbf{7 7 5}$ & 694 & 530 & 505 & 353 & 473 & 446 \\
Área basal total & $\mathbf{2 1}$ & 32 & 19 & 19 & 18 & 19 & 25 \\
Coeficiente mezcla & $\mathbf{7 , 6}$ & 4,7 & 4,4 & 4,2 & 3,9 & 3,8 & 3,1 \\
Índice de Shanon & $\mathbf{5 , 1 1}$ & 6,1 & 5,9 & 6,1 & 5,5 & 5,7 & 6,3 \\
Índice de Fisher & $\mathbf{3 1}$ & 54,2 & 44,6 & 51,1 & 39 & 54 & 75,8 \\
Altitud (msnm) & $\mathbf{1 0 9 0}$ & 2100 & 2275 & 1150 & 1075 & 1150 & 1500 \\
\hline
\end{tabular}


Tabla 3 - Familias frecuentes y especiosas registradas en los bosques de Selva Central, en parcelas de una hectárea ubicados entre 990 y 2275 msnm.

Table 3 - Most frequent families registered in the Upper Amazonian forests of Central Peru, in one hectare plots located between 990 and 2275 masl.

\begin{tabular}{lcccccccc}
\hline Familias & P-ST & P-PL & P-PR & P-GC & P-GL & P-MTL & P-CPH & prom \\
\hline Lauraceae & $\mathbf{1 1}$ & 29 & 7 & 14 & 10 & 4 & 18 & 13,29 \\
Moraceae & $\mathbf{2 0}$ & 12 & 3 & 12 & 10 & 6 & 11 & 10,57 \\
Rubiaceae & $\mathbf{3}$ & 8 & 10 & 7 & 4 & 10 & 4 & 6,57 \\
Melastomataceae & 7 & 11 & 14 & 4 & 0 & 5 & 3 & 6,29 \\
Urticaceae & $\mathbf{4}$ & 7 & 7 & 6 & 8 & 6 & 5 & 6,14 \\
Leguminosae & $\mathbf{1 1}$ & 0 & 0 & 7 & 9 & 11 & 3 & 5,86 \\
Euphorbiaceae & $\mathbf{7}$ & 4 & 8 & 4 & 3 & 9 & 4 & 5,57 \\
Meliaceae & $\mathbf{2}$ & 2 & 2 & 4 & 5 & 5 & 7 & 3,86 \\
Clusiaceae & $\mathbf{2}$ & 6 & 3 & 4 & 2 & 3 & 2 & 3,14 \\
Malvaceae & $\mathbf{1}$ & 0 & 0 & 6 & 5 & 5 & 3 & 2,86 \\
Myrtaceae & $\mathbf{2}$ & 8 & 5 & 2 & 0 & 2 & 1 & 2,86 \\
Myrsinaceae & $\mathbf{2}$ & 5 & 6 & 2 & 0 & 2 & 2 & 2,71 \\
Annonaceae & $\mathbf{2}$ & 5 & 1 & 1 & 3 & 1 & 2 & 2,14 \\
Sapindaceae & $\mathbf{1}$ & 2 & 3 & 4 & 2 & 1 & 1 & 2 \\
Cyatheaceae & $\mathbf{1}$ & 3 & 6 & 1 & 0 & 1 & 0 & 1,71 \\
\hline
\end{tabular}

Tabla 4 - Géneros frecuentes registrados en los bosques de Selva Central, en parcelas de una hectárea ubicados entre 990 y 2275 msnm.

Table 4 - Most frequent genera registered in the Upper Amazonian forests of Central Peru, in one hectare plots located between 990 and 2275 masl.

\begin{tabular}{lcccccccc}
\hline Géneros & P-ST & P-PL & P-PR & P-GC & P-GL & P-MTL & P-CPH & prom. \\
\hline Ocotea & 8 & 4 & 2 & 7 & 2 & 3 & 22 & 6,86 \\
Ficus & 10 & 10 & 2 & 4 & 3 & 4 & 6 & 5,57 \\
Nectandra & 1 & 7 & 3 & 3 & 2 & 2 & 13 & 4,43 \\
Inga & 5 & 3 & 0 & 6 & 8 & 6 & 2 & 4,29 \\
Miconia & 6 & 9 & 9 & 0 & 0 & 3 & 2 & 4,14 \\
Cecropia & 2 & 4 & 5 & 4 & 4 & 3 & 1 & 3,29 \\
Myrsine & 2 & 4 & 3 & 1 & 0 & 2 & 0 & 1,71 \\
Allophylus & 1 & 1 & 2 & 3 & 2 & 1 & 1 & 1,57 \\
Alchornea & 2 & 1 & 1 & 1 & 1 & 2 & 1 & 1,29 \\
Virola & 3 & 0 & 0 & 1 & 1 & 1 & 3 & 1,29 \\
Aspidosperma & 1 & 0 & 0 & 3 & 2 & 2 & 0 & 1,14 \\
Trichilia & 1 & 0 & 0 & 3 & 2 & 1 & 1 & 1,14 \\
Pourouma & 2 & 0 & 0 & 1 & 2 & 1 & 1 & 1,00 \\
Clarisia & 2 & 0 & 0 & 2 & 2 & 1 & 0 & 1,00 \\
Pseudolmedia & 2 & 1 & 0 & 1 & 1 & 0 & 2 & 1,00 \\
Calyptranthes & 1 & 3 & 3 & 0 & 0 & 0 & 0 & 1,00 \\
Piper & 0 & 2 & 2 & 0 & 1 & 0 & 2 & 1,00 \\
\hline
\end{tabular}


(15), las cinco especies poseen el $32.2 \%$ de la abundancia total del bosque de cima de colina (Reynel \& Antón 2004). También Ladenbergia oblongifolia (Humb. ex Mutis) L. Andersson (56), Cecropia polystachya Trécul (30), Sapium glandulosum (L.) Morong (28), Iriartea deltoidea Ruiz \& Pav. (23), Zapoteca amazonica (Benth.) H.M. Hern (16), Macbrideina peruviana Standl.y Persea caerulea (Ruiz \& Pav.) Mez (15), las siete especies poseen el $38.6 \%$ de la abundancia total para un bosque de ladera (Antón \& Reynel 2004). Para un bosque de ladera a $1075 \mathrm{~m}$ las especies más abundantes fueron Otoba parvifolia (Markgr.) A.H. Gentry (35), Nectandra pulverulenta (29), B. costaricensis y S. exorrhiza (24) y P. laevis (14), las cinco especies poseen el $35.6 \%$ de la abundancia total (Fig. 5 y anexo 1). En la zona de estudio $G$. hyposericea, P. minor, A. glandulosa, $V$. venulosa, H. sylvestris y C. arborea, poseen el $29.4 \%$ de la abundancia total. Hacemos notar que no se comparte ninguna especie abundante y frecuente en el piso premontano y montano a pesar que entre algunas parcelas la distancia en línea recta no sobrepasa los dos kilómetros (Fig. 1).

La presencia de dos especies nuevas, Protium y Magnolia, revela que son necesarios más inventarios florísticos en nuestro país, para comprender mejor los patrones de composición florística. Protium ha sido considerado exclusivamente amazónico con 29 especies registradas en Perú entre 0-500 msnm y cuatro especies registradas entre los $0-1000$

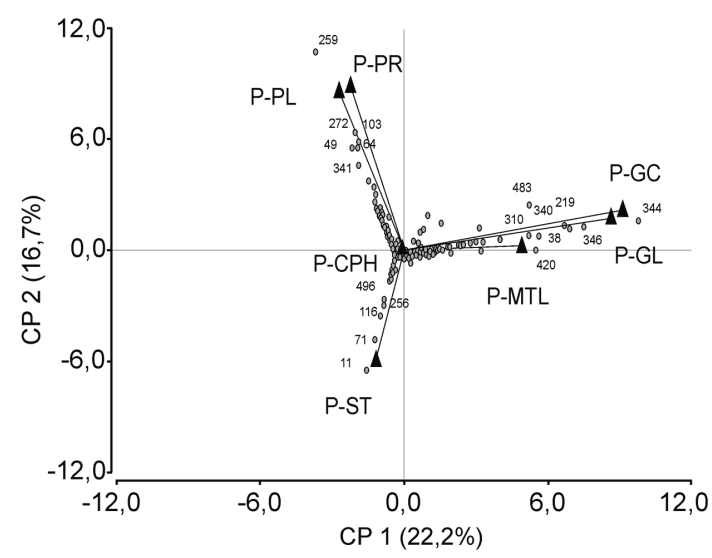

Figura 5 - Diagrama de dispersión de especies con datos de abundancia total de siete parcelas de 01 ha localizadas en La Merced, San Ramón y Satipo.

Figure 5 - Species diagram of scatter with data of total abundance in seven 1.0 ha forest plots in La Merced, San Ramon and Satipo. m (Brako \& Zarucchi 1993; Ulloa et al. 2004). Esto refleja que el conocimiento de los bosques neotropicales sigue siendo extremadamente pobre.

El índice de diversidad de Fisher en el presente estudio fue de 31. Estudios en selva central reportan que el índice de Fisher oscila entre 39 y 75.8 (Tab. 2). Todos los valores de los índices de diversidad analizados son mayores que la muestra de estudio. Note que el índice de Fisher permite hacer comparaciones relativamente fieles del nivel de diversidad de especies entre sitios, que dada una misma área, varían en términos de abundancia. A diferencia de la mayoría de los índices que se suelen utilizar para cuantificar la diversidad (Magurran 1988). El índice de Fisher establece de manera explícita, que la diversidad (riqueza de especies) depende del número de individuos muestreados. Así desde el punto de vista matemático, este índice "controla" y "elimina", por el tamaño de la muestra (en este caso número de individuos), el efecto positivo que tiene la abundancia sobre la diversidad, lo que permite determinar si una parcela de bosque es realmente más diversa que otra (Berry 2002).

\section{Del análisis estructural}

En la Amazonía peruana, Pitman et al. (2001), reportan para nueve parcelas permanentes con un rango de 465-724 individuos por parcela con un promedio de $29.2 \mathrm{~m}^{2}$ de área basal. Para la Amazonía ecuatoriana. Así mismo, estudios realizados en Selva Central (Chanchamayo y San Ramón), con la misma metodología reportan valores de densidad que oscilan entre 353 y 694 individuos y, un área basal que oscilan entre $19 \mathrm{y}$ 32 m² (Antón \& Reynel 2003; La Torre et al. 2007). La muestra en estudio registró 775 individuos, posee mayor densidad que las muestras de bosque al suroeste de la Amazonía Peruana. Así mismo, es mayor que las muestras de selva central (Antón \& Reynel 2003; La Torre 2007). Sin embargo, el área basal de $21.60 \mathrm{~m}^{2}$, solo es mayor que la $\mathrm{P}-\mathrm{GC}$, P-GL, P-MCT y P-PR (Tab. 2).

\section{Análisis comparativo de fragmentos} de bosque en el valle de Chanchamayo La ordenación MDS de los sitios analizados tomando como referencia datos de presencia/ ausencia, abundancia total y abundancia con log transformado, reconocieron cuatro grupos distintos de ensamblajes de especies de árboles (1) los bosques de Santa Teresa, (2) los cedros de Pampa Hermosa, (3) La Génova y la microcuenca 
El Tirol y (4) los bosques de Pichita que podrían reflejar una menor o mayor combinación de gradientes altitudinales en el valle de chanchamayo. La separación notable de las P-ST y la P-CPH, se debió a la exclusividad de las especies. Por ejemplo, 78 especies son exclusivas de la P-ST. Así mismo, la P-CPH presentó 91 especies exclusivas, mientras que 33 especies fueron exclusivas en las parcelas P-PL, P-PR y 10 especies para la P-GL, P-GC y P-MTL. Mientras que la ordenación MDS de géneros con datos de abundancia total o con $\log$ transformado, reconocieron cinco grupos de ensamblajes de árboles: Santa Teresa, La Génova, microcuenca El Tirol, Cedros de Pampa Hermosa y Pichita.

Bosques por debajo de los 1500 presentan afinidades con los bosques amazónicos, particularmente por P. laevis, Clarisia biflora Ruiz \& Pav., C. racemosa Ruiz \& Pav. y B. costaricensis (Moraceae), L. oblongifolia (Rubiceae), S. exorrhiza (Arecaceae), Garcinia madruno (Kunth) Hammel (Clusiaceae) y O. parvifolia (Myristicaceae) que pueden ser especies conspicuas en bosques maduros de tierras bajas (Antón \& Reynel 2004). Por otro lado, un típico estrato montano de altas elevaciones (mayor de $1500 \mathrm{~m}$ ) puede estar inferido por Weinmannia lechleriana Engl. (Cunoniaceae), Hyeronima asperifolia Pax \& K. Hoffm. y $H$. oblonga (Tull) Müll. Arg (Euphorbiaceae) (Antón y Reynel 2004) y la alta densidad de individuos de Melastomataceae en los bosques de Pichita (P-PL y P-PR).

\section{Conclusiones}

La muestra de bosque de Santa Teresa, presenta 102 especies, 67 géneros y 37 familias. La diversidad alfa es moderada en contraste con otras muestras de bosque de selva central y amazonía baja. Se asume que la naturaleza transicional, la carencia de lianas y la tala selectiva son características que evidencian la baja diversidad de la zona de estudio.

En Selva central, los bosques premontanos guardan afinidades florísticas entre ellos y con los de amazonia baja, pero hay diferencias marcas con el bosque montano por la mayor presencia de familias y géneros altoandinos. Las familias Lauraceae, Moraceae, Rubiaceae, Melastomataceae, Urticaceae y Leguminosae, así como, los géneros Ocotea, Ficus, Nectandra, Inga y Miconia son los más ricos en especies en los bosques de selva central del Perú.
El conocimiento sobre la flora arbórea de selva central sigue siendo extremadamente pobre. Es clara la necesidad de continuar haciendo muestreos a gran escala y de uniformizar metodologías para analizar en forma adecuada los patrones de diversidad y composición florística.

\section{Agradecimientos}

Al Sr. A. Daza y D. Elera, por su colaboración en el inventario y colecciones dendrológicas. A los Ing. Juan Anahui y Carlos Chuquicaja por ofrecernos todas las facilidades en el Fundo Santa Teresa. A todos los estudiantes que participaron en el trabajo de campo y de herbario. A J. Saito y J. Rivera por los mapas digitales.

\section{Bibliografía}

Antón, B.D. \& Reynel, C. 2004. Diversidad y composición florística de la flora arbórea en un área de ladera de colinas en bosque premontanos: Microcuenca de Tirol, valle de Chanchamayo, 1000-1500 msnm. In: Antón, D. \& Reynel, C. (eds.). Relictos de bosque de excepcional diversidad en los Andes centrales de Perú. Perú Darwin Project-PBR - APRODESUniversidad Nacional Agraria La Molina (UNALM), Lima. Pp. 221-262.

APG II. 2003. An update of the Angiosperm Phlogeny Group, classification for the orders and families of Flowering plants: Botanical Journal of the Linnean Society 141: 399-436.

Berry, P.E. 2002. Diversidad y endemismo en los bosques neotropicales de bajura. In: Guariguata, M.R. \& Kattan, G.H. (eds.). Ecología de Bosques Neotropicales. Libro Universitario Regional, Cartago. Pp. 83-96.

Brack, E. A.\& C. Mendiola V. 2000. Ecología del Perú. Bruño, Lima. 495p.

Brako J. \& Zarucchi J.L. 1993. Catalogue of the flowering plants and gymnosperms of Peru. Monographs in Systematic Botany. Vol. 45. The Missouri Botanical Garden, St. Louis. 1286p.

Bridson, D. \& Forman, L. 1999. The herbarium handbook. $3^{\text {rd }}$ ed. Royal Botanic Gardens, Kew. 334p.

Brown, K.S.Jr. 1977. Centros de evolução, refugios quaternaries e conservacão de patrimonies genéticos na região neotropical: patroes de diferenacão em lihomiinae (Lepidoptera: Nymphalidae). Acta Amazonica 7: 75-137

Bussmann, R.W. 2001. The montane forests of Reserva Biológica San Francisco (ZamoraChinchipe, Ecuador) vegetation zonation and natural regeneration. Die Erde 132: 9-25

Caro, S.; Reynel, C. \& Antón, D. 2004. Diversidad y composición de la flora arbórea en un área de ladera de colinas en bosque premontano: Fundo Génova- 
UNALM, Valle de Chanchamayo, 1000-1500 msnm. In: Antón, D. \& Reynel, C. (eds.). Relictos de bosque de excepcional diversidad en los Andes centrales de Perú. Perú Darwin Project-PBR APRODES-Universidad Nacional Agraria La Molina (UNALM), Lima. Pp. 187-220.

Casas, H. 1989. Productividad y estructura de costos de extracción y transporte de madera rolliza en el valle de Chanchamayo. Tesis de Graduación. Universidad Nacional Agraria La Molina, Lima. 144p.

Churchill, S.P.; Balslev H.; Forero, E. \& Luteyn, J.L. (eds.). 1995. Biodiversity and conservation of neotropical montane forests. New York Botanical Garden Press, New York.

Clarke, K.R. \& Gorley, R. 2001. PRIMER v5. User Manual/Tutorial. Primer-E, Plymouth. 91p.

Cleef, A.M.; Rangel, O.C.; Van der Hammen, T. \& Jaramillo, R. 1984. La vegetación de las selvas del transecto Buritaca. In: Van der Hammen, T. \& Ruíz, P.M. (eds.). La Sierra Nevada de Santa Marta (Colombia), Transecto Buritaca-La Cumbre. Studies on Tropical Andean Ecosystems 2. J. Cramer, Berlin. Pp. 267-406

Curtis, J.T. \& Mcintosh, R.P. 1951. An unpland forest continum in the prairie forest border region of Wisconsin. Ecology 32: 476-496.

Di Rienzo, J.A.; Casanoves, F.; Balzarini, M.G.; Gonzalez, L.; Tablada, M. \& Robledo, C.W. InfoStat versión 2012. Grupo InfoStat, FCA, Universidad Nacional de Córdoba. Disponível em $<$ http://www. infostat.com.ar>. Acesso en Oct 2013.

Fisher, R.A.; Corbet y A.S. \& Williams, C.B. 1943. The relation between the number of species and the number of individuals in a random sample of an animal population. Journal of Animal Ecology 12:42-58.

Fjeldså, J.; Lambin, E. \& Mertens, B. 1999. Correlations between endemism and local ecoclimatic stability documented by comparing Andean bird distributions and remotely sensed land surface data. Ecography 22: 63-78

Gentry, A.H. 1982a. Patterns of neotropical plant species diversity. Evolutionary Biology 15: 1-84.

Gentry A.H. 1988a. Changes in plant community diversity and floristic composition on environmental and geographical gradients. Annals Missouri Botanical Garden 75: 1-34.

Gentry A.H. 1988b. Tree species richness of upper Amazonian forests. Proceedings of the National Academy of Sciences of the United States of America 85: 156-159.

Gentry, A.H. 1989. Northwest South America (Colombia, Ecuador and Peru). In: Campbell, D. \& Hammond, H.D. (eds.). Floristic inventory of tropical forests. The New York Botanical Garden, New York. Pp. 392-400.
Gentry, A.H. 1992. Diversity and floristic composition of Andean forest of Peru and adjacent contries: implications of their conservation. In: Young, K.R. \& Valencia, N. (eds.). Biogeografía, ecología y conservación del bosque montano en el Perú. Memorias del Museo de Historia Natural 21. Universidad Nacional Mayor de San Marcos, Lima. Pp. 11-29.

Gentry, A. \& Ortiz, R. 1993. Patrones de composición florística en la Amazonía peruana. Kalliola, R.; Puhakka, M. \& Danyoy, W. (eds.). Amazonia peruana vegetación húmeda subtropical en el llano subandino. Proyecto Amazonia Universidad de Turku (PAUT) and Oficina Nacional de Evaluación de Recursos Naturales (ONERN), Jyväskylä. Pp. 155-166.

Gentry A.H. 1995. Patterns of diversity and floristic composition in neotropical rain forest. In: Churchill, S.P.; Balslev, H.; Forero, E. \& Luteyn, J.L. (eds.). Niodiversity and conservation of neotropical montane forests. New York Botanical Garden Press, New York. Pp. 103-126.

Hamilton, L.S.; Juvik, J.O. \& Skatena, F.N. (eds.). 1995. Tropical montane cloud forests. Ecological Studies Vol. 110. Springer Verlag, Nueva York. 407p.

Holdridge, L.R. 1978. Ecología basada en zonas de vida. Instituto Interamericano de Ciencias Agrícolas, San José. 216p.

Huamantupa, Ch. I. 2010. Inusual riqueza, composición y estructura arbórea en el bosque de tierra firme del Pongo Qoñec, Sur Oriente peruano. Revista Peruana de Biología 17: 167-171.

INRENA 1995. Mapa ecológico del Perú. Mapa y guía explicative. Instituto Nacional de recursos Naturales, Lima. 220p.

Kapos, V.; Rhind, J.; Edwards, M. \& Price, M.F. 2000. Developing a map of the world's mountain forest. In: Price, M.F. \& Butts, N. (eds.). Forest in sustainable mountain development: a state of knowledge report for 2000. Commonwealth Agricultural Bureau (CAB) International, Wallingford.

La Torre-Cuadros, M.A. 2003. Composición florística y diversidad en el bosque relicto los Cedros de Pampa Hermosa (Chanchamayo, Junín) e implicancias para su conservación. Tesis Magister Scientiae. Universidad Nacional Agraria La Molina, Lima. $141 \mathrm{p}$.

La Torre-Cuadros, M.A.; Herrando-Pérez, S. \& Young, K. 2007. Diversity and structural patterns for tropical montane ad premontane forest of central Peru, With an assessment of the use of higher-taxon surrogacy. Biodivers Conserv. 16: 2965-2988.

Magurran, A.E. 1987. Ecological diversity and its measurement. Princeton University Press, Princeton. 179 p.

Ministerio del Ambiente (MINAM). 2011. El Perú de los bosques. Lima. 140p. 
Mori, S.; Boom, B.; Carvalino, M.A. \& Santos, S. 1983. Ecological importance of Myrtaceae in an Eastern Brazilian we forest. Biotropica 15: 68-70

Oliveira, A.A. \& Mori, S.A. 1999. A central Amazonian terra firme forest. I. High species richness on poor soils. Biodiversity and Conservation 8: 1219-1259.

ONERN. 1976. Mapa ecológico del Perú. Guía explicativa. Oficina Nacional de Evaluación de Recursos Naturales (ONERN), Lima. 151p.

Phillips O.L.; Hall, P.; Gentry, A.H. et al. 1994. Dynamics and species richness of tropical rain forests. Proceedings of the National Academy of Sciences of the United States of America 91: 2805-2809.

Phillips, O. \& Baker, T. 2006. Manual de campo para la remedición y establecimiento de parcelas. RAINFOR. Proyecto PAN AMAZONIA. 18p.

Pitman N.C.A.; Terborg, J.; Silman, M.R. et al. 2001. Dominance and distribution of tree species in upper Amazonian terra firme forests. Ecology 82: 2101-2117.

Pitman, N.C.A.; Terborg, J.; Silman, M.R.; Nuñez, P. \& W.A. Palacios. 2001. Dominance and distribution of tree species in upper Amazonian terra firme forest. Ecology 82: 2101-2117

Prance, G.T. 1973. Phytogeographic support for the theory of Pleistocene Forest refuges in the Amazon Basin, based on evidence from distribution patterns in Caryocaraceae, Chrysobalanaceae, Dichapetalaceae and Lecythidaceae. Acta Amazonica 3: 5-28

Rangel, J.O. 1991. Ecología de ecosistemas Andinos en Colombia. Ph.D. Dissertation. Universidad de Amsterdam, Amsterdam. 392p.

Reynel, R.C. \& Antón, B.D. 2004. Diversidad y composición de la flora arbórea en un área de cumbre de colinas en bosque premontano: Fundo Génova-UNALM, valle de Chanchamayo 10001500 msnm. In: Antón, D. \& Reynel, C. (eds.). Relictos de bosque de excepcional diversidad en los Andes centrales de Perú. Perú Darwin ProjectPBR - APRODES-Universidad Nacional Agraria La Molina (UNALM), Lima. Pp. 143-186

Reynel, R.C. \& Antón, B.D. 2004. Diversidad y composición de la flora arbórea en un área ribereña de bosque montano: Pichita, valle de Chanchamayo 2000-2500 msnm. In: Antón, D. \& Reynel, C. (eds.). Relictos de bosque de excepcional diversidad en los Andes centrales de Perú. Perú Darwin ProjectPBR - APRODES-Universidad Nacional Agraria La Molina (UNALM), Lima. Pp. 99-142.

Reynel, R.C. \& Honorio, E. 2004. Diversidad y composición de la flora arbórea en un área de ladera de bosque montano: Pichita, valle de Chanchamayo 2000-2500 msnm. In: Antón, D. \& Reynel, C. (eds.). Relictos de bosque de excepcional diversidad en los Andes centrales de Perú. Perú Darwin ProjectPBR - APRODES-Universidad Nacional Agraria La Molina (UNALM), Lima. Pp. 45-98.

ter Steege, H.; Sabatier, S.; Castellanos, H.H.; van Andel, T.; Duivenvoorden, J.; Oliveira, A.A.; Ek, R.C.; Lilwah, R.; Maas, P.J.M. \& Mori, S.A. 2000. An analysis of the floristic composition and diversity of Amazonian forests including those of the Guiana Shield. Journal of Tropical Ecology 16: 801-828.

Vásquez, M.R.; Rojas, G.R.; Monteagudo, M.A.; Van der Werff, H. \& Ortiz, R. 2005. Flora vascular de la selva central del Perú: una aproximación de la composición florística de tres Áreas Naturales Protegidas. Arnaldoa 12: 112-125. 


\section{Patrones de diversidad y composición florística de parcelas de evaluación permanente en la selva central de Perú}

Diversity patterns and floristic composition of permanent evaluative plots in the Peruvian central forest

José Luis Marcelo-Peña \& Carlos Reynel Rodriguez

Apéndice - Lista de especies registradas en siete parcela de 1 ha localizadas en La Merced, San Ramón y Satipo. Appendix - List of species recorded in seven 1.0 ha forest plots in La Merced, San Ramon and Satipo.

\begin{tabular}{|c|c|c|c|c|c|c|c|c|c|}
\hline $\mathbf{N}^{\circ}$ & Especies & Familias & P-ST & P-PL & P-PR P & P-GC & P-GL & P-MTL & P-CPH \\
\hline 1 & Ruellia sp.1 & ACANTHACEAE & 0 & 0 & 0 & 0 & 0 & 0 & 7 \\
\hline 2 & Astronium graveolens Jacq. & ANACARDIACEAE & 3 & 0 & 0 & 0 & 0 & 0 & 0 \\
\hline 3 & Mauria heterophylla Kunth & ANACARDIACEAE & 0 & 0 & 0 & 6 & 3 & 9 & 0 \\
\hline 4 & Tapirira guianensis Aubl. & ANACARDIACEAE & 0 & 0 & 0 & 3 & 0 & 3 & 1 \\
\hline 5 & Annona ambotay Aubl. & ANNONACEAE & 0 & 2 & 0 & 0 & 0 & 0 & 0 \\
\hline 6 & $\begin{array}{l}\text { Annona cordifolia (Szyszyl.) Poepp. ex Maas } \\
\text { \& Westra }\end{array}$ & ANNONACEAE & 0 & 3 & 0 & 0 & 0 & 0 & 0 \\
\hline 7 & Annona montana Macfad. & ANNONACEAE & 0 & 0 & 0 & 0 & 0 & 1 & 0 \\
\hline 8 & Cymbopetalum sp.1 & ANNONACEAE & 0 & 0 & 0 & 0 & 0 & 0 & 1 \\
\hline 9 & Cymbopetalum sp. 2 & ANNONACEAE & 0 & 0 & 0 & 0 & 3 & 0 & 0 \\
\hline 10 & Guatteria dielsiana R.E. Fr. & ANNONACEAE & 0 & 4 & 0 & 0 & 0 & 0 & 0 \\
\hline 11 & Guatteria hyposericea Diels & ANNONACEAE & 119 & 0 & 0 & 0 & 0 & 0 & 0 \\
\hline 12 & Guatteria trichoclonia Diels aff. & ANNONACEAE & 2 & 0 & 0 & 0 & 0 & 0 & 0 \\
\hline 13 & Guatteria $\mathrm{sp} .1$ & ANNONACEAE & 0 & 1 & 1 & 0 & 0 & 0 & 0 \\
\hline 14 & Oxandra acuminata Diels & ANNONACEAE & 0 & 0 & 0 & 0 & 5 & 0 & 0 \\
\hline 15 & Oxandra sp.1 & ANNONACEAE & 0 & 0 & 0 & 0 & 1 & 0 & 0 \\
\hline 16 & Rollinia cuspidata Mart. & ANNONACEAE & 0 & 1 & 0 & 0 & 0 & 0 & 0 \\
\hline 17 & Rollinia sp. & ANNONACEAE & 0 & 0 & 0 & 0 & 0 & 0 & 2 \\
\hline 18 & Rollinia sp.1 & ANNONACEAE & 0 & 0 & 0 & 1 & 0 & 0 & 0 \\
\hline 19 & Aspidosperma capitatum L.O.Williams & APOCYNACEAE & 0 & 0 & 0 & 0 & 0 & 1 & 0 \\
\hline 20 & Aspidosperma cylindrocarpon Müll. Arg. & APOCYNACEAE & 0 & 0 & 0 & 12 & 1 & 0 & 0 \\
\hline 21 & Aspidosperma macrocarpon Mart. & APOCYNACEAE & 1 & 0 & 0 & 0 & 0 & 0 & 0 \\
\hline 22 & Aspidosperma parvifolium A. DC. & APOCYNACEAE & 0 & 0 & 0 & 2 & 0 & 0 & 0 \\
\hline 23 & Aspidosperma polyneuron Müll. Arg. & APOCYNACEAE & 0 & 0 & 0 & 1 & 0 & 1 & 0 \\
\hline 24 & Aspidosperma sp. 1 & APOCYNACEAE & 0 & 0 & 0 & 0 & 1 & 0 & 0 \\
\hline 25 & Hancornia sp.1 & APOCYNACEAE & 1 & 0 & 0 & 0 & 0 & 0 & 0 \\
\hline
\end{tabular}




\begin{tabular}{|c|c|c|c|c|c|c|c|c|c|}
\hline $\mathbf{N}^{0}$ & Especies & Familias & P-ST & P-PL & P-PR & P-GC & P-GL & P-MTL & P-CPH \\
\hline 26 & Lacmellea sp. 1 & APOCYNACEAE & 1 & 0 & 0 & 0 & 0 & 0 & 0 \\
\hline 27 & Symphonia globulifera L. f. & APOCYNACEAE & 2 & 0 & 0 & 0 & 0 & 0 & 0 \\
\hline 28 & Ilex sp.1 & AQUIFOLIACEAE & 0 & 0 & 1 & 0 & 0 & 0 & 0 \\
\hline 29 & Ilex sp.2 & AQUIFOLIACEAE & 0 & 0 & 1 & 0 & 0 & 0 & 0 \\
\hline 30 & Ceroxylon vogelianum (Engel) H.Wendl. & ARACACEAE & 0 & 6 & 0 & 0 & 0 & 0 & 0 \\
\hline 31 & Dendropanax arboreus (L.) Decne. \& Planch. & ARALIACEAE & 0 & 2 & 0 & 0 & 0 & 0 & 0 \\
\hline 32 & Dendropanax sp. & ARALIACEAE & 0 & 0 & 0 & 0 & 0 & 0 & 4 \\
\hline 33 & Dendropanax sp.1 & ARALIACEAE & 0 & 0 & 0 & 2 & 1 & 0 & 0 \\
\hline 34 & Oreopanax sp. 1 & ARALIACEAE & 0 & 0 & 1 & 4 & 1 & 0 & 0 \\
\hline 35 & Oreopanax sp.2 & ARALIACEAE & 0 & 0 & 0 & 0 & 2 & 0 & 0 \\
\hline 36 & $\begin{array}{l}\text { Schefflera morototoni (Aubl.) Maguire, } \\
\text { Steyerm. \& Frodin }\end{array}$ & ARALIACEAE & 1 & 0 & 0 & 0 & 0 & 1 & 0 \\
\hline 37 & Iriartea deltoidea Ruiz \& Pav. & ARECACEAE & 0 & 0 & 0 & 1 & 0 & 23 & 0 \\
\hline 38 & Socratea exorrhiza (Mart.) H. Wendl. & ARECACEAE & 1 & 0 & 0 & 11 & 24 & 0 & 0 \\
\hline 39 & $\begin{array}{l}\text { Jacaranda glabra (A. DC.) Bureau \& } \\
\text { Schumann }\end{array}$ & BIGNONIACEAE & 19 & 0 & 0 & 0 & 0 & 0 & 0 \\
\hline 40 & Bixa urucurana Willd. & BIXACEAE & 0 & 0 & 0 & 0 & 0 & 1 & 0 \\
\hline 41 & Ceiba insignis (H. B. K.) Gibbs \& Semir & BOMBACACEAE & 1 & 0 & 0 & 0 & 0 & 6 & 0 \\
\hline 42 & Ceiba pentandra (L.) Gaertn. & BOMBACACEAE & 0 & 0 & 0 & 0 & 0 & 0 & 1 \\
\hline 43 & Ochroma pyramidale (Cav. ex Lam.) Urb. & BOMBACACEAE & 0 & 0 & 0 & 2 & 0 & 13 & 0 \\
\hline 44 & $\begin{array}{l}\text { Pseudobombax munguba (Mart. \& Zucc.) } \\
\text { Dugand }\end{array}$ & BOMBACACEAE & 0 & 0 & 0 & 2 & 3 & 1 & 0 \\
\hline 45 & Quararibea sp. & BOMBACACEAE & 0 & 0 & 0 & 0 & 0 & 0 & 2 \\
\hline 46 & Cordia sp.1 & BORAGINACEAE & 0 & 1 & 0 & 0 & 0 & 0 & 0 \\
\hline 47 & Brunellia dulcis J.F. Macbr. & BRUNELLIACEAE & 0 & 0 & 18 & 0 & 0 & 0 & 0 \\
\hline 48 & Protium sp. 1 & BURSERACEAE & 0 & 2 & 0 & 0 & 0 & 0 & 0 \\
\hline 49 & Protium sp. nov. & BURSERACEAE & 0 & 44 & 1 & 0 & 0 & 0 & 0 \\
\hline 50 & Protium tenuifolium (Engl.) Engl. & BURSERACEAE & 2 & 0 & 0 & 0 & 0 & 0 & 0 \\
\hline 51 & Trattinnickia lawrancei Standley & BURSERACEAE & 25 & 0 & 0 & 0 & 0 & 0 & 0 \\
\hline 52 & Capparis schunkei J.F. Macbr. & CAPPARACEAE & 3 & 0 & 0 & 0 & 0 & 0 & 0 \\
\hline 53 & Sambucus peruviana Kunth & CAPRIFOLIACEAE & 0 & 3 & 7 & 0 & 0 & 0 & 0 \\
\hline 54 & Jacaratia sp. & CARICACEAE & 0 & 0 & 0 & 0 & 0 & 0 & 6 \\
\hline 55 & Caryocar glabrum (Aubl.) Pers. & CARYOCARIACEAE & 7 & 0 & 0 & 0 & 0 & 0 & 0 \\
\hline 56 & Cecropia ficifolia Warburg ex Snethlage & CECROPIACEAE & 1 & 0 & 0 & 0 & 0 & 0 & 0 \\
\hline 57 & Cecropia hispidissima Cuatrec. & CECROPIACEAE & 0 & 0 & 0 & 0 & 2 & 0 & 0 \\
\hline 58 & Cecropia membranacea Trécul & CECROPIACEAE & 0 & 0 & 0 & 1 & 4 & 0 & 0 \\
\hline 59 & Cecropia polystachia Trécul & CECROPIACEAE & 0 & 0 & 0 & 1 & 3 & 30 & 0 \\
\hline
\end{tabular}




\begin{tabular}{|c|c|c|c|c|c|c|c|c|c|}
\hline $\mathbf{N}^{\circ}$ & Especies & Familias & P-ST & P-PL & P-PR & P-GC & P-GL & P-MTL I & P-CPH \\
\hline 60 & Cecropia sciadophylla C. Martius & CECROPIACEAE & 1 & 0 & 0 & 0 & 0 & 0 & 0 \\
\hline 61 & Cecropia sp. & CECROPIACEAE & 0 & 0 & 0 & 0 & 0 & 0 & 4 \\
\hline 62 & Cecropia sp.1 & CECROPIACEAE & 0 & 5 & 0 & 3 & 1 & 1 & 0 \\
\hline 63 & Cecropia $\mathrm{sp} .2$ & CECROPIACEAE & 0 & 5 & 0 & 1 & 0 & 1 & 0 \\
\hline 64 & Cecropia sp.3 & CECROPIACEAE & 0 & 11 & 24 & 0 & 0 & 0 & 0 \\
\hline 65 & Cecropia sp.4 & CECROPIACEAE & 0 & 4 & 4 & 0 & 0 & 0 & 0 \\
\hline 66 & Cecropia sp.5 & CECROPIACEAE & 0 & 0 & 3 & 0 & 0 & 0 & 0 \\
\hline 67 & Coussapoa manuensis C.C. Berg & CECROPIACEAE & 0 & 0 & 0 & 7 & 0 & 1 & 0 \\
\hline 68 & Coussapoa villosa Poepp. \& Endl & CECROPIACEAE & 0 & 1 & 0 & 0 & 0 & 0 & 0 \\
\hline 69 & Pourouma cecropiifolia Mart. & CECROPIACEAE & 0 & 0 & 0 & 7 & 6 & 1 & 0 \\
\hline 70 & Pourouma guianensis Aublet & CECROPIACEAE & 3 & 0 & 0 & 0 & 0 & 0 & 0 \\
\hline 71 & Pourouma minor Benoist & CECROPIACEAE & 87 & 0 & 0 & 0 & 0 & 0 & 0 \\
\hline 72 & Pourouma sp. & CECROPIACEAE & 0 & 0 & 0 & 0 & 0 & 0 & 1 \\
\hline 73 & Pourouma sp.1 & CECROPIACEAE & 0 & 0 & 0 & 0 & 2 & 0 & 0 \\
\hline 74 & Maytenus macrocarpa (Ruiz \& Pav.) Briq. & CELASTRACEAE & 0 & 0 & 0 & 2 & 0 & 0 & 0 \\
\hline 75 & Maytenus sp. & CELASTRACEAE & 0 & 0 & 0 & 0 & 0 & 0 & 2 \\
\hline 76 & Hedyosmum sp.1 & CHLORANTHACEAE & 0 & 0 & 1 & 0 & 0 & 0 & 0 \\
\hline 77 & Hedyosmum sp. 2 & CHLORANTHACEAE & 0 & 0 & 2 & 0 & 0 & 0 & 0 \\
\hline 78 & $\begin{array}{l}\text { Couepia chrysocalyx (Poepp.) Benth. ex } \\
\text { Hook.f. }\end{array}$ & CHRYSOBALANACEAE & 0 & 1 & 0 & 0 & 0 & 0 & 0 \\
\hline 79 & Hirtella triandra Sw. & CHRYSOBALANACEAE & 0 & 0 & 0 & 0 & 0 & 6 & 0 \\
\hline 80 & Hirtella sp.1 & CHRYSOBALANACEAE & 0 & 0 & 0 & 0 & 0 & 1 & 0 \\
\hline 81 & $\begin{array}{l}\text { Licania octandra (Hoffmanns. ex Roem. \& } \\
\text { Schult.) Kuntze }\end{array}$ & CHRYSOBALANACEAE & 1 & 0 & 0 & 0 & 0 & 0 & 0 \\
\hline 82 & Clethra peruviana Szyszyl. & CLETHRACEAE & 0 & 1 & 0 & 0 & 0 & 0 & 0 \\
\hline 83 & Clethra revoluta (Ruiz \& Pav.) Spreng. & CLETHRACEAE & 0 & 1 & 0 & 0 & 0 & 0 & 0 \\
\hline 84 & Clethra sp.1 & CLETHRACEAE & 0 & 1 & 0 & 0 & 0 & 0 & 0 \\
\hline 85 & Calophyllum brasiliense Cambess. & CLUSIACEAE & 0 & 0 & 0 & 2 & 0 & 0 & 0 \\
\hline 86 & Clusia ducu Benth. & CLUSIACEAE & 0 & 2 & 0 & 0 & 0 & 0 & 0 \\
\hline 87 & Clusia elliptica Kunth & CLUSIACEAE & 0 & 6 & 3 & 0 & 0 & 0 & 0 \\
\hline 88 & Clusia longistyla Cuatrec. & CLUSIACEAE & 0 & 0 & 2 & 0 & 0 & 0 & 0 \\
\hline 89 & Clusia sp. & CLUSIACEAE & 0 & 0 & 0 & 0 & 0 & 0 & 1 \\
\hline 90 & Clusia sp.1 & CLUSIACEAE & 0 & 0 & 0 & 0 & 0 & 1 & 0 \\
\hline 91 & Clusia sp.2 & CLUSIACEAE & 0 & 0 & 2 & 0 & 0 & 0 & 0 \\
\hline 92 & Garcinia macrophylla Mart. & CLUSIACEAE & 0 & 0 & 0 & 3 & 5 & 0 & 1 \\
\hline 93 & Garcinia madruno (Kunth) Hammel & CLUSIACEAE & 0 & 0 & 0 & 13 & 3 & 0 & 0 \\
\hline 94 & Marila laxiflora Rusby & CLUSIACEAE & 0 & 0 & 0 & 2 & 0 & 5 & 0 \\
\hline
\end{tabular}




\begin{tabular}{|c|c|c|c|c|c|c|c|c|c|}
\hline $\mathbf{N}^{\circ}$ & Especies & Familias & P-ST & P-PL & P-PR & P-GC & P-GL & P-MTL F & P-CPH \\
\hline 95 & Marila sp.1 & CLUSIACEAE & 0 & 0 & 0 & 0 & 0 & 3 & 0 \\
\hline 96 & Tovomita sp.1 & CLUSIACEAE & 0 & 1 & 0 & 0 & 0 & 0 & 0 \\
\hline 97 & Vismia baccifera $($ L.) Triana \& Planch. & CLUSIACEAE & 0 & 1 & 0 & 0 & 0 & 0 & 0 \\
\hline 98 & Buchenavia sp. & COMBRETACEAE & 0 & 0 & 0 & 0 & 0 & 0 & 2 \\
\hline 99 & Buchenavia sp.1 & COMBRETACEAE & 0 & 0 & 0 & 1 & 0 & 0 & 0 \\
\hline 100 & Terminalia amazonia (J.F. Gmel.) Exell & COMBRETACEAE & 0 & 0 & 0 & 8 & 0 & 3 & 0 \\
\hline 101 & Terminalia sp. & COMBRETACEAE & 0 & 0 & 0 & 0 & 0 & 0 & 1 \\
\hline 102 & Weinmannia latifolia C. Presl & CUNONIACEAE & 0 & 0 & 1 & 0 & 0 & 0 & 0 \\
\hline 103 & Weinmannia lechleriana Engl. & CUNONIACEAE & 0 & 0 & 33 & 0 & 0 & 0 & 0 \\
\hline 104 & Weinmannia lentiscifolia C. Presl & CUNONIACEAE & 0 & 10 & 2 & 0 & 0 & 0 & 0 \\
\hline 105 & Weinmannia sp.1 & CUNONIACEAE & 0 & 0 & 1 & 0 & 0 & 0 & 0 \\
\hline 106 & Alsophylla cuspidata (Kunze) D.S. Conant & CYATHEACEAE & 0 & 0 & 1 & 0 & 0 & 0 & 0 \\
\hline 107 & Cyathea sp. 1 & CYATHEACEAE & 6 & 2 & 1 & 1 & 0 & 7 & 0 \\
\hline 108 & Cyathea sp.2 & CYATHEACEAE & 0 & 3 & 0 & 0 & 0 & 0 & 0 \\
\hline 109 & Cyathea sp.3 & CYATHEACEAE & 0 & 2 & 0 & 0 & 0 & 0 & 0 \\
\hline 110 & $\begin{array}{l}\text { Sphaeropteris quindiuensis (H. Karst.) R.M. } \\
\text { Tryon }\end{array}$ & CYATHEACEAE & 0 & 0 & 13 & 0 & 0 & 0 & 0 \\
\hline 111 & Sloanea rufa Planchon ex Bentham aff. & ELAEOCARPACEAE & 1 & 0 & 0 & 0 & 0 & 1 & 0 \\
\hline 112 & Sloanea sp.1 & ELAEOCARPACEAE & 0 & 0 & 0 & 0 & 0 & 4 & 0 \\
\hline 113 & Erythroxyllum gracilipes Peyritsch & ERYTHROXYLLACEAE & 1 & 0 & 0 & 0 & 0 & 0 & 0 \\
\hline 114 & Acalypha sp.1 & EUPHORBIACEAE & 0 & 0 & 18 & 0 & 0 & 0 & 0 \\
\hline 115 & Alchornea brittonii Secco & EUPHORBIACEAE & 0 & 8 & 6 & 0 & 0 & 0 & 0 \\
\hline 116 & Alchornea glandulosa Poeppig & EUPHORBIACEAE & 62 & 0 & 0 & 0 & 0 & 0 & 0 \\
\hline 117 & Alchornea triplinervia (Sprengel) Muel. Arg. & EUPHORBIACEAE & 3 & 0 & 0 & 1 & 1 & 7 & 3 \\
\hline 118 & Aparisthmium cordatum (A.Juss.) Baill. & EUPHORBIACEAE & 0 & 0 & 0 & 0 & 0 & 0 & 2 \\
\hline 119 & Croton draconoides Müll. Arg. & EUPHORBIACEAE & 0 & 0 & 0 & 0 & 0 & 1 & 0 \\
\hline 120 & Croton sp. 1 & EUPHORBIACEAE & 0 & 0 & 14 & 0 & 0 & 0 & 0 \\
\hline 121 & Drypetes amazonica Steyerm. & EUPHORBIACEAE & 0 & 0 & 0 & 4 & 4 & 0 & 0 \\
\hline 122 & $\begin{array}{l}\text { Hevea guianensis var. lutea (Spruce ex } \\
\text { Bentham) Ducke \& Schultes }\end{array}$ & EUPHORBIACEAE & 5 & 0 & 0 & 0 & 0 & 0 & 0 \\
\hline 123 & Hyeronima alchornoides Ruiz \& Pav. & EUPHORBIACEAE & 0 & 0 & 0 & 0 & 0 & 1 & 0 \\
\hline 124 & Hyeronima asperifolia Pax \& K. Hoffm & EUPHORBIACEAE & 0 & 0 & 20 & 0 & 0 & 0 & 0 \\
\hline 125 & Hyeronima oblonga Pax \& K. Hoffm & EUPHORBIACEAE & 0 & 16 & 0 & 0 & 0 & 0 & 0 \\
\hline 126 & Hyeronima sp.1 & EUPHORBIACEAE & 0 & 0 & 0 & 0 & 0 & 2 & 0 \\
\hline 127 & Mabea sp.1 & EUPHORBIACEAE & 0 & 0 & 1 & 0 & 0 & 0 & 0 \\
\hline 128 & Margaritaria nobilis L.f. & EUPHORBIACEAE & 0 & 0 & 0 & 1 & 3 & 5 & 0 \\
\hline 129 & Pausandra trianae (Muell. Arg.) Baillon & EUPHORBIACEAE & 1 & 0 & 0 & 0 & 0 & 0 & 0 \\
\hline
\end{tabular}




\begin{tabular}{|c|c|c|c|c|c|c|c|c|c|}
\hline $\mathbf{N}^{\mathbf{0}}$ & Especies & Familias & P-ST & P-PL & P-PR I & P-GC & P-GL & P-MTL I & P-CPH \\
\hline 130 & Pseudosenefeldera inclinata (Müll.Arg.) Esser & EUPHORBIACEAE & 4 & 0 & 0 & 0 & 0 & 0 & 0 \\
\hline 131 & Richeria grandis Vahl & EUPHORBIACEAE & 0 & 0 & 0 & 0 & 0 & 1 & 0 \\
\hline 132 & Sapium glandulosum (L.) Morong & EUPHORBIACEAE & 0 & 4 & 3 & 6 & 0 & 28 & 0 \\
\hline 133 & Sapium marmierii Huber & EUPHORBIACEAE & 1 & 0 & 0 & 0 & 0 & 0 & 0 \\
\hline 134 & Sapium sp. & EUPHORBIACEAE & 0 & 0 & 0 & 0 & 0 & 0 & 1 \\
\hline 135 & Tetrorchidium macrophyllum Müll. Arg. & EUPHORBIACEAE & 0 & 0 & 1 & 0 & 0 & 0 & 0 \\
\hline 136 & Tetrorchidium rubrivenium Poepp. & EUPHORBIACEAE & 0 & 0 & 0 & 0 & 0 & 0 & 1 \\
\hline 137 & Tetrorchidium sp. & EUPHORBIACEAE & 0 & 0 & 0 & 0 & 0 & 1 & 0 \\
\hline 138 & Casearia arborea (Rich.) Urb. & FLACOURTIACEAE & 24 & 0 & 0 & 0 & 0 & 0 & 0 \\
\hline 139 & Casearia javitensis Kunth & FLACOURTIACEAE & 0 & 0 & 0 & 0 & 0 & 1 & 0 \\
\hline 140 & Casearia sp.1 & FLACOURTIACEAE & 0 & 1 & 1 & 0 & 0 & 0 & 0 \\
\hline 141 & Hasseltia floribunda Kunth & FLACOURTIACEAE & 0 & 0 & 0 & 1 & 1 & 0 & 0 \\
\hline 142 & Homalium racemosum Jacq. & FLACOURTIACEAE & 0 & 1 & 3 & 0 & 0 & 0 & 0 \\
\hline 143 & Mayna sp. 1 & FLACOURTIACEAE & 1 & 0 & 0 & 0 & 0 & 0 & 0 \\
\hline 144 & Cheiloclinium cognatum (Miers) A.C. Sm. aff. & HIPPOCRATEACEAE & 1 & 0 & 0 & 0 & 0 & 0 & 0 \\
\hline 145 & Hydrangea sp.1 & HYDRANGEACEAE & 0 & 1 & 0 & 0 & 0 & 0 & 0 \\
\hline 146 & Dioscophora guianensis Miers & ICACINACEAE & 2 & 0 & 0 & 0 & 0 & 0 & 0 \\
\hline 147 & Juglans neotropica Diels & JUGLANDACEAE & 0 & 0 & 0 & 0 & 0 & 0 & 1 \\
\hline 148 & Lacistema agregatum (P.J.Bergius) Rusby & LACISTEMATACEAE & 0 & 0 & 0 & 0 & 0 & 1 & 0 \\
\hline 149 & Lozania mutisiana Schult. & LACISTEMATACEAE & 0 & 0 & 9 & 0 & 0 & 0 & 0 \\
\hline 150 & Aniba megaphylla $\mathrm{Mez}$ & LAURACEAE & 0 & 1 & 0 & 0 & 0 & 0 & 0 \\
\hline 151 & $\begin{array}{l}\text { Aniba robusta (Klotzsch \& H.Karst. ex Meisn.) } \\
\text { Mez }\end{array}$ & LAURACEAE & 0 & 1 & 0 & 0 & 0 & 0 & 0 \\
\hline 152 & Aniba sp.1 & LAURACEAE & 0 & 0 & 1 & 0 & 0 & 0 & 0 \\
\hline 153 & Aniba sp.2 & LAURACEAE & 0 & 1 & 0 & 0 & 0 & 0 & 0 \\
\hline 154 & Aniba sp. 3 & LAURACEAE & 0 & 7 & 0 & 0 & 0 & 0 & 0 \\
\hline 155 & Aniba sp.4 & LAURACEAE & 0 & 1 & 0 & 0 & 0 & 0 & 0 \\
\hline 156 & Aniba sp.5 & LAURACEAE & 0 & 2 & 0 & 0 & 0 & 0 & 0 \\
\hline 157 & Beilschmiedia sulcata (Ruiz \& Pav.) Kosterm. & LAURACEAE & 0 & 13 & 0 & 0 & 0 & 0 & 0 \\
\hline 158 & Beilschmiedia sp.1 & LAURACEAE & 0 & 0 & 0 & 0 & 4 & 0 & 0 \\
\hline 159 & $\begin{array}{l}\text { Cinnamomum triplenerve (Ruiz \& Pav.) } \\
\text { Kosterm. }\end{array}$ & LAURACEAE & 0 & 2 & 0 & 0 & 0 & 7 & 0 \\
\hline 160 & Cinnamomum sp.1 & LAURACEAE & 0 & 1 & 0 & 0 & 0 & 0 & 0 \\
\hline 161 & Endlicheria sericea Nees & LAURACEAE & 0 & 0 & 0 & 1 & 0 & 0 & 0 \\
\hline 162 & Endlicheria sp.1 & LAURACEAE & 0 & 1 & 0 & 0 & 0 & 0 & 2 \\
\hline 163 & Endlicheria $\mathrm{sp} .2$ & LAURACEAE & 0 & 0 & 0 & 0 & 0 & 0 & 3 \\
\hline 164 & Licaria pucheri (Ruiz \& Pav.) Kosterm. & LAURACEAE & 0 & 0 & 0 & 1 & 4 & 0 & 0 \\
\hline
\end{tabular}




\begin{tabular}{|c|c|c|c|c|c|c|c|c|c|}
\hline $\mathbf{N}^{o}$ & Especies & Familias & P-ST & P-PL & P-PR & P-GC & P-GL & P-MTL & P-CPH \\
\hline 165 & Licaria triandra (Sw.) Kosterm. & LAURACEAE & 0 & 0 & 0 & 1 & 0 & 0 & 0 \\
\hline 166 & Mezilaurus sp.1 & LAURACEAE & 0 & 0 & 0 & 2 & 6 & 0 & 0 \\
\hline 167 & Nectandra cuspidata Nees & LAURACEAE & 4 & 0 & 0 & 0 & 0 & 0 & 0 \\
\hline 168 & Nectandra longifolia (Ruiz \& Pav.) Nees & LAURACEAE & 0 & 1 & 0 & 2 & 1 & 4 & 4 \\
\hline 169 & Nectandra pulverulenta Nees & LAURACEAE & 0 & 0 & 0 & 7 & 29 & 4 & 1 \\
\hline 170 & Nectandra reticulata $\mathrm{Mez}$ & LAURACEAE & 0 & 0 & 1 & 0 & 0 & 0 & 4 \\
\hline 171 & Nectandra sp. & LAURACEAE & 0 & 14 & 0 & 0 & 0 & 0 & 0 \\
\hline 172 & Nectandra sp. 1 & LAURACEAE & 0 & 0 & 12 & 0 & 0 & 0 & 4 \\
\hline 173 & Nectandra sp.2 & LAURACEAE & 0 & 0 & 11 & 1 & 0 & 0 & 1 \\
\hline 174 & Nectandra sp.3 & LAURACEAE & 0 & 9 & 0 & 0 & 0 & 0 & 1 \\
\hline 175 & Nectandra sp.4 & LAURACEAE & 0 & 8 & 0 & 0 & 0 & 0 & 1 \\
\hline 176 & Nectandra sp.5 & LAURACEAE & 0 & 1 & 0 & 0 & 0 & 0 & 3 \\
\hline 177 & Nectandra sp.6 & LAURACEAE & 0 & 1 & 0 & 0 & 0 & 0 & 1 \\
\hline 178 & Nectandra sp.7 & LAURACEAE & 0 & 0 & 0 & 0 & 0 & 0 & 1 \\
\hline 179 & Nectandra sp.8 & LAURACEAE & 0 & 0 & 0 & 0 & 0 & 0 & 1 \\
\hline 180 & Nectandra sp.9 & LAURACEAE & 0 & 0 & 0 & 0 & 0 & 0 & 3 \\
\hline 181 & Nectandra utilis Rohwer & LAURACEAE & 0 & 9 & 0 & 0 & 0 & 0 & 0 \\
\hline 182 & Ocotea aciphylla (Nees) Mez & LAURACEAE & 1 & 0 & 0 & 0 & 4 & 1 & 1 \\
\hline 183 & Ocotea argyrophylla Ducke & LAURACEAE & 0 & 0 & 0 & 1 & 0 & 0 & 0 \\
\hline 184 & Ocotea bofo Kunth & LAURACEAE & 2 & 0 & 0 & 0 & 0 & 0 & 0 \\
\hline 185 & Ocotea cernua (Nees) Mez aff. & LAURACEAE & 1 & 0 & 0 & 2 & 0 & 1 & 0 \\
\hline 186 & Ocotea forma reflexa & LAURACEAE & 3 & 0 & 0 & 0 & 0 & 0 & 0 \\
\hline 187 & Ocotea javitensis (Kunth) Pittier & LAURACEAE & 0 & 1 & 0 & 0 & 0 & 0 & 0 \\
\hline 188 & Ocotea leucoxylon (Swartz) de Lanessan aff. & LAURACEAE & 1 & 0 & 0 & 0 & 0 & 0 & 0 \\
\hline 189 & Ocotea oblonga (Meisn.) Mez & LAURACEAE & 1 & 0 & 0 & 0 & 0 & 0 & 0 \\
\hline 190 & Ocotea obovata (R. \& P.) Mez & LAURACEAE & 6 & 13 & 0 & 1 & 0 & 0 & 0 \\
\hline 191 & Ocotea ovalifolia (Ruiz \& Pav.) Mez & LAURACEAE & 0 & 0 & 0 & 2 & 2 & 1 & 0 \\
\hline 192 & Ocotea sp. 1 & LAURACEAE & 0 & 8 & 0 & 0 & 0 & 0 & 2 \\
\hline 193 & Ocotea $\mathrm{sp} .2$ & LAURACEAE & 0 & 19 & 1 & 0 & 0 & 0 & 3 \\
\hline 194 & Ocotea sp. 3 & LAURACEAE & 0 & 0 & 13 & 0 & 0 & 0 & 1 \\
\hline 195 & Ocotea sp.4 & LAURACEAE & 0 & 0 & 0 & 0 & 0 & 0 & 1 \\
\hline 196 & Ocotea sp.5 & LAURACEAE & 0 & 0 & 0 & 1 & 0 & 0 & 3 \\
\hline 197 & Ocotea sp.6 & LAURACEAE & 0 & 0 & 0 & 3 & 0 & 0 & 4 \\
\hline 198 & Ocotea sp.7 & LAURACEAE & 0 & 0 & 0 & 0 & 0 & 0 & 6 \\
\hline 199 & Ocotea $\mathrm{sp} .8$ & LAURACEAE & 0 & 0 & 0 & 0 & 0 & 0 & 3 \\
\hline 200 & Ocotea sp.9 & LAURACEAE & 0 & 0 & 0 & 0 & 0 & 0 & 4 \\
\hline
\end{tabular}




\begin{tabular}{|c|c|c|c|c|c|c|c|c|}
\hline $\mathbf{N}^{0}$ Especies & Familias & P-ST & P-PL & P-PR & P-GC & P-GL & P-MTL & P-CPH \\
\hline 201 Persea americana Mill. & LAURACEAE & 0 & 1 & 0 & 0 & 0 & 15 & 0 \\
\hline 202 Persea sp. & LAURACEAE & 0 & 0 & 0 & 0 & 0 & 0 & 2 \\
\hline 203 Persea sp. 1 & LAURACEAE & 0 & 0 & 0 & 0 & 1 & 0 & 0 \\
\hline 204 Persea sp. 2 & LAURACEAE & 0 & 0 & 0 & 0 & 0 & 1 & 0 \\
\hline 205 Pleurothyrium cuneifolium Nees & LAURACEAE & 0 & 1 & 0 & 0 & 0 & 0 & 0 \\
\hline 206 Rhodostemonodaphnekunthiana (Nees) Rohwer & LAURACEAE & 2 & 0 & 0 & 0 & 0 & 0 & 0 \\
\hline 207 Cariniana estrellensis (Raddi) Kuntze & LECYTHIDACEAE & 0 & 0 & 0 & 1 & 0 & 0 & 0 \\
\hline 208 Eschweilera coriacea (A. CD.) S. Mori & LECYTHIDACEAE & 7 & 0 & 0 & 0 & 0 & 0 & 0 \\
\hline 209 Eschweilera sp.1 & LECYTHIDACEAE & 0 & 0 & 0 & 0 & 0 & 1 & 0 \\
\hline 210 Albizia carbonaria Britton & LEGUMINOSAE & 0 & 0 & 0 & 0 & 0 & 5 & 0 \\
\hline 211 Bauhinia sp.1 & LEGUMINOSAE & 0 & 0 & 0 & 6 & 0 & 1 & 0 \\
\hline 212 Calliandra sp. 1 & LEGUMINOSAE & 0 & 0 & 0 & 0 & 0 & 1 & 0 \\
\hline 213 Cassia grandis L. f. aff. & LEGUMINOSAE & 15 & 0 & 0 & 0 & 0 & 1 & 0 \\
\hline 214 Cedrelinga cateniformis (Ducke) Ducke & LEGUMINOSAE & 15 & 0 & 0 & 0 & 0 & 0 & 0 \\
\hline 215 Enterolobium sp. 1 & LEGUMINOSAE & 0 & 0 & 0 & 0 & 0 & 1 & 0 \\
\hline 216 Erythrina sp. 1 & LEGUMINOSAE & 0 & 0 & 0 & 1 & 0 & 0 & 0 \\
\hline 217 Inga alba (Sw.) Willd. & LEGUMINOSAE & 0 & 0 & 0 & 0 & 3 & 0 & 0 \\
\hline 218 Inga chartacea Poepp. & LEGUMINOSAE & 0 & 0 & 0 & 2 & 0 & 0 & 0 \\
\hline 219 Inga cinnamoena Spruce ex Benth. & LEGUMINOSAE & 0 & 0 & 0 & 34 & 0 & 0 & 0 \\
\hline 220 Inga edulis Mart. & LEGUMINOSAE & 0 & 0 & 0 & 0 & 0 & 1 & 0 \\
\hline 221 Inga klugii Stanley ex J. F. Macbride aff. & LEGUMINOSAE & 1 & 0 & 0 & 0 & 0 & 0 & 0 \\
\hline 222 Inga macrophylla Humb. \& Bonpl. ex Willd. & LEGUMINOSAE & 0 & 0 & 0 & 0 & 0 & 0 & 1 \\
\hline 223 Inga marginata Willd. & LEGUMINOSAE & 0 & 1 & 0 & 0 & 2 & 0 & 0 \\
\hline 224 Inga ruiziana $\mathrm{G}$. Don & LEGUMINOSAE & 1 & 0 & 0 & 0 & 0 & 0 & 0 \\
\hline 225 Inga sapindoides Willd. & LEGUMINOSAE & 0 & 0 & 0 & 0 & 1 & 0 & 0 \\
\hline 226 Inga setosa G. Don & LEGUMINOSAE & 0 & 6 & 3 & 4 & 6 & 2 & 0 \\
\hline 227 Inga striata Benth. & LEGUMINOSAE & 0 & 12 & 0 & 0 & 0 & 0 & 0 \\
\hline 228 Inga thibaudiana DC. & LEGUMINOSAE & 10 & 0 & 0 & 3 & 0 & 1 & 0 \\
\hline 229 Inga forma asimetrica & LEGUMINOSAE & 7 & 0 & 0 & 0 & 0 & 0 & 0 \\
\hline 230 Inga forma levemente alada & LEGUMINOSAE & 3 & 0 & 0 & 0 & 0 & 0 & 0 \\
\hline 231 Inga sp.1 & LEGUMINOSAE & 0 & 0 & 0 & 6 & 2 & 1 & 0 \\
\hline 232 Inga sp.2 & LEGUMINOSAE & 0 & 0 & 0 & 0 & 1 & 1 & 0 \\
\hline 233 Inga sp.3 & LEGUMINOSAE & 0 & 0 & 0 & 0 & 1 & 2 & 0 \\
\hline 234 Inga sp.4 & LEGUMINOSAE & 0 & 0 & 1 & 0 & 1 & 0 & 0 \\
\hline 235 Inga sp.5 & LEGUMINOSAE & 0 & 0 & 0 & 0 & 1 & 0 & 0 \\
\hline 236 Lecointea peruviana Standl. ex J.F. Macbr. & LEGUMINOSAE & 0 & 0 & 0 & 0 & 0 & 1 & 1 \\
\hline
\end{tabular}




\begin{tabular}{|c|c|c|c|c|c|c|c|c|c|}
\hline $\mathbf{N}^{o}$ & Especies & Familias & P-ST & P-PL & P-PR F & P-GC & P-GL & P-MTL & P-CPH \\
\hline 237 & Machaerium millei Standl. & LEGUMINOSAE & 0 & 0 & 0 & 0 & 0 & 1 & 0 \\
\hline 238 & Machaerium sp.1 & LEGUMINOSAE & 0 & 0 & 0 & 0 & 0 & 3 & 0 \\
\hline 239 & Machaerium sp.2 & LEGUMINOSAE & 0 & 0 & 0 & 3 & 1 & 1 & 0 \\
\hline 240 & Myroxylon balsamum (L.) Harms & LEGUMINOSAE & 0 & 0 & 0 & 0 & 0 & 2 & 0 \\
\hline 241 & $\begin{array}{l}\text { Ormosia coccinea var. subsimplex (Spruce ex } \\
\text { Bentham) Rudd }\end{array}$ & LEGUMINOSAE & 1 & 0 & 0 & 0 & 0 & 0 & 0 \\
\hline 242 & Parkia multijuga Benth. aff. & LEGUMINOSAE & 7 & 0 & 0 & 0 & 0 & 0 & 0 \\
\hline 243 & Stryphnodendron forma ferruginea & LEGUMINOSAE & 4 & 0 & 0 & 0 & 0 & 0 & 0 \\
\hline 244 & $\begin{array}{l}\text { Tachigali chrysophylla (Poepp.) Zarucchi \& } \\
\text { Herend. }\end{array}$ & LEGUMINOSAE & 0 & 0 & 0 & 0 & 0 & 1 & 0 \\
\hline 245 & $\begin{array}{l}\text { Tachigali peruviana (Dwyer) Zarucchi \& } \\
\text { Herendeen }\end{array}$ & LEGUMINOSAE & 1 & 0 & 0 & 0 & 0 & 0 & 0 \\
\hline 246 & Tachigali sp.1 & LEGUMINOSAE & 0 & 8 & 0 & 0 & 0 & 1 & 0 \\
\hline 247 & Zapoteca amazonica (Benth.) H.M. Hern. & LEGUMINOSAE & 0 & 0 & 0 & 0 & 0 & 17 & 0 \\
\hline 248 & Magnolia sp. nova & MAGNOLIACEAE & 0 & 9 & 6 & 0 & 0 & 0 & 0 \\
\hline 249 & Buchonsia sp.1 & MALPHIGIACEAE & 0 & 0 & 0 & 6 & 0 & 0 & 0 \\
\hline 250 & Byrsonima spicata (Cav.) Rich. ex Kunth i & MALPHIGIACEAE & 9 & 0 & 0 & 0 & 0 & 0 & 0 \\
\hline 251 & Bellucia pentamera Naudin & MELASTOMATACEAE & 5 & 0 & 0 & 0 & 0 & 0 & 0 \\
\hline 252 & Bellucia sp.1 & MELASTOMATACEAE & 0 & 0 & 0 & 0 & 0 & 3 & 0 \\
\hline 253 & Blakea sp.1 & MELASTOMATACEAE & 0 & 0 & 1 & 0 & 0 & 0 & 0 \\
\hline 254 & Graffenrieda intermedia Triana & MELASTOMATACEAE & 0 & 1 & 7 & 0 & 0 & 0 & 0 \\
\hline 255 & Graffenrieda sp.1 & MELASTOMATACEAE & 0 & 0 & 1 & 0 & 0 & 1 & 0 \\
\hline 256 & Henrietella sylvestris Gleason & MELASTOMATACEAE & 46 & 0 & 0 & 0 & 0 & 0 & 0 \\
\hline 257 & Miconia aulocalix Mart. & MELASTOMATACEAE & 2 & 0 & 0 & 0 & 0 & 0 & 0 \\
\hline 258 & Miconia aurea (D. Don) Naudin & MELASTOMATACEAE & 2 & 0 & 0 & 0 & 0 & 0 & 0 \\
\hline 259 & Miconia aureoides Cogn. & MELASTOMATACEAE & 0 & 66 & 14 & 0 & 0 & 0 & 0 \\
\hline 260 & Miconia barbeyana Cogn. & MELASTOMATACEAE & 18 & 0 & 0 & 0 & 0 & 0 & 0 \\
\hline 261 & Miconia calophylla (D. Don) Triana & MELASTOMATACEAE & 0 & 2 & 0 & 0 & 0 & 0 & 0 \\
\hline 262 & Miconia calvescens DC. & MELASTOMATACEAE & 0 & 0 & 0 & 0 & 0 & 1 & 0 \\
\hline 263 & Miconia denticulata Naudin & MELASTOMATACEAE & 0 & 17 & 4 & 0 & 0 & 0 & 0 \\
\hline 264 & Miconia eriocalyx Naudin & MELASTOMATACEAE & 0 & 3 & 3 & 0 & 0 & 0 & 0 \\
\hline 265 & Miconia lamprophylla Triana aff. & MELASTOMATACEAE & 4 & 0 & 0 & 0 & 0 & 0 & 0 \\
\hline 266 & Miconia theaezans (Bonpl.) Cogn. & MELASTOMATACEAE & 0 & 1 & 1 & 0 & 0 & 0 & 0 \\
\hline 267 & Miconia forma envés dorado & MELASTOMATACEAE & 1 & 0 & 0 & 0 & 0 & 0 & 0 \\
\hline 268 & Miconia forma olor a canela & MELASTOMATACEAE & 11 & 0 & 0 & 0 & 0 & 0 & 0 \\
\hline 269 & Miconia sp.1 & MELASTOMATACEAE & 0 & 8 & 0 & 0 & 0 & 1 & 2 \\
\hline 270 & Miconia sp.2 & MELASTOMATACEAE & 0 & 1 & 0 & 0 & 0 & 2 & 2 \\
\hline 271 & Miconia sp.3 & MELASTOMATACEAE & 0 & 1 & 1 & 0 & 0 & 0 & 0 \\
\hline
\end{tabular}




\begin{tabular}{|c|c|c|c|c|c|c|c|c|}
\hline $\mathbf{N}^{0}$ Especies & Familias & P-ST & P-PL & P-PR I & P-GC & P-GL & P-MTL & P-CPH \\
\hline 272 Miconia sp.4 & MELASTOMATACEAE & 0 & 0 & 36 & 0 & 0 & 0 & 0 \\
\hline 273 Miconia sp.6 & MELASTOMATACEAE & 0 & 0 & 2 & 0 & 0 & 0 & 0 \\
\hline 274 Miconia sp.7 & MELASTOMATACEAE & 0 & 0 & 2 & 0 & 0 & 0 & 0 \\
\hline 275 Mouriri sp. & MELASTOMATACEAE & 0 & 0 & 0 & 0 & 0 & 0 & 1 \\
\hline 276 Mouriri sp. 1 & MELASTOMATACEAE & 0 & 8 & 0 & 6 & 0 & 1 & 0 \\
\hline 277 Mouriri sp. 2 & MELASTOMATACEAE & 0 & 6 & 0 & 1 & 0 & 1 & 0 \\
\hline 278 Tibouchina sp.1 & MELASTOMATACEAE & 0 & 0 & 0 & 0 & 0 & 5 & 0 \\
\hline 279 Tibouchina sp.2 & MELASTOMATACEAE & 0 & 0 & 0 & 0 & 0 & 1 & 0 \\
\hline 280 Cabralea canjerana (Vell.) Mart. & MELIACEAE & 11 & 0 & 0 & 0 & 0 & 0 & 0 \\
\hline 281 Cedrela angustifolia Sessé \& Moc. ex DC. & MELIACEAE & 0 & 0 & 0 & 0 & 0 & 0 & 9 \\
\hline 282 Cedrela fissilis Vell. & MELIACEAE & 0 & 0 & 0 & 0 & 0 & 2 & 0 \\
\hline 283 Cedrela odorata L. & MELIACEAE & 0 & 0 & 0 & 0 & 1 & 1 & 0 \\
\hline 284 Guarea glabra Vahl & MELIACEAE & 0 & 0 & 0 & 0 & 0 & 0 & 1 \\
\hline 285 Guarea guidonia (L.) Sleumer & MELIACEAE & 0 & 0 & 0 & 0 & 0 & 0 & 3 \\
\hline 286 Guarea kunthiana A. Juss. & MELIACEAE & 0 & 4 & 7 & 6 & 2 & 2 & 7 \\
\hline 287 Guarea macrophylla Vahl & MELIACEAE & 0 & 0 & 0 & 0 & 0 & 0 & 1 \\
\hline 288 Guarea sp. & MELIACEAE & 0 & 0 & 0 & 0 & 0 & 0 & 9 \\
\hline 289 Rudgea amazonica Müll. Arg. & MELIACEAE & 0 & 2 & 0 & 0 & 0 & 0 & 0 \\
\hline 290 Rudgea sp. & MELIACEAE & 0 & 9 & 3 & 0 & 0 & 0 & 0 \\
\hline 291 Trichilia adolfii Harms & MELIACEAE & 0 & 0 & 0 & 0 & 2 & 0 & 0 \\
\hline 292 Trichilia maynasiana C. DC. & MELIACEAE & 0 & 0 & 0 & 0 & 0 & 1 & 2 \\
\hline 293 Trichilia pleeana (A. Juss.) C. DC. & MELIACEAE & 0 & 0 & 0 & 8 & 1 & 9 & 0 \\
\hline 294 Trichilia septentrionalis C. DC. & MELIACEAE & 1 & 0 & 0 & 0 & 0 & 0 & 0 \\
\hline 295 Trichilia sp.2 & MELIACEAE & 0 & 0 & 0 & 1 & 0 & 0 & 0 \\
\hline 296 Trichilia sp. 3 & MELIACEAE & 0 & 0 & 0 & 1 & 0 & 0 & 0 \\
\hline 297 Meliosma glabrata (Liebm.) Urb. & MELIOSMACEAE & 0 & 1 & 0 & 0 & 0 & 0 & 0 \\
\hline 298 Meliosma herbertii Rolfe & MELIOSMACEAE & 0 & 0 & 5 & 0 & 0 & 0 & 0 \\
\hline 299 Meliosma sp. & MELIOSMACEAE & 0 & 0 & 0 & 0 & 0 & 0 & 1 \\
\hline 300 Meliosma sp. 1 & MELIOSMACEAE & 0 & 2 & 0 & 1 & 0 & 0 & 0 \\
\hline 301 Meliosma sp.2 & MELIOSMACEAE & 0 & 0 & 0 & 0 & 0 & 0 & 1 \\
\hline 302 Mollinedia sp. 1 & MONIMIACEAE & 0 & 7 & 1 & 0 & 0 & 0 & 0 \\
\hline 303 Mollinedia sp.2 & MONIMIACEAE & 0 & 3 & 2 & 0 & 0 & 0 & 0 \\
\hline 304 Siparuna sp.1 & MONIMIACEAE & 0 & 0 & 2 & 0 & 0 & 0 & 0 \\
\hline $\begin{array}{l}305 \text { Batocarpus costaricensis Standl. \& L.O. } \\
\text { William }\end{array}$ & MORACEAE & 0 & 0 & 0 & 19 & 24 & 0 & 0 \\
\hline 306 Brosimum guianense (Aubl.) Huber & MORACEAE & 0 & 0 & 0 & 1 & 0 & 0 & 0 \\
\hline
\end{tabular}




\begin{tabular}{|c|c|c|c|c|c|c|c|c|c|}
\hline $\mathbf{N}^{\circ}$ & Especies & Familias & P-ST & P-PL & P-PR & P-GC & P-GL & P-MTL F & $\mathrm{P}-\mathrm{CPH}$ \\
\hline 307 & Brosimum utile (Kunth) Oken ex J. Presl & MORACEAE & 5 & 0 & 0 & 0 & 0 & 0 & 0 \\
\hline 308 & Brosimum sp. & MORACEAE & 0 & 0 & 0 & 0 & 0 & 0 & 1 \\
\hline 309 & Castilla ulei Warburg & MORACEAE & 1 & 0 & 0 & 0 & 0 & 0 & 0 \\
\hline 310 & Clarisia biflora Ruiz \& Pav. & MORACEAE & 1 & 0 & 0 & 13 & 12 & 4 & 0 \\
\hline 311 & Clarisia racemosa R.\& P. & MORACEAE & 1 & 0 & 0 & 10 & 11 & 0 & 0 \\
\hline 312 & Ficus americanaAubl. & MORACEAE & 1 & 0 & 0 & 0 & 1 & 0 & 1 \\
\hline 313 & $\begin{array}{l}\text { Ficus americana subsp.guianensis (Desv. ex } \\
\text { Ham.) C.C.Berg }\end{array}$ & MORACEAE & 0 & 1 & 0 & 1 & 0 & 0 & 0 \\
\hline 314 & Ficus cervantesiana Dugand & MORACEAE & 1 & 0 & 0 & 0 & 0 & 0 & 0 \\
\hline 315 & Ficus citrifolia Mill. & MORACEAE & 0 & 0 & 0 & 0 & 0 & 1 & 0 \\
\hline 316 & Ficus coerulescens (Rusby) Rossberg & MORACEAE & 0 & 1 & 0 & 0 & 0 & 0 & 0 \\
\hline 317 & Ficus crassiuscula Warb.ex Standl. & MORACEAE & 1 & 3 & 3 & 0 & 0 & 0 & 0 \\
\hline 318 & Ficus cutrecasana Dugand & MORACEAE & 1 & 5 & 0 & 1 & 0 & 0 & 1 \\
\hline 319 & Ficus gigantosyce Dugand & MORACEAE & 0 & 1 & 0 & 0 & 0 & 0 & 0 \\
\hline 320 & Ficus gomelleira Kunth \& C.D. Bouché & MORACEAE & 1 & 0 & 0 & 0 & 0 & 0 & 0 \\
\hline 321 & Ficus insipida Willd. & MORACEAE & 1 & 0 & 0 & 0 & 0 & 0 & 0 \\
\hline 322 & Ficus macbridei Standl. & MORACEAE & 0 & 2 & 0 & 0 & 0 & 1 & 2 \\
\hline 323 & Ficus maxima Mill. & MORACEAE & 2 & 1 & 0 & 1 & 0 & 1 & 3 \\
\hline 324 & Ficus mutisii Dugand & MORACEAE & 0 & 1 & 0 & 0 & 0 & 0 & 0 \\
\hline 325 & Ficus nymphaeifolia Mill. & MORACEAE & 0 & 0 & 0 & 0 & 0 & 0 & 1 \\
\hline 326 & Ficus obtusifolia Kunth & MORACEAE & 0 & 0 & 0 & 0 & 1 & 0 & 0 \\
\hline 327 & Ficus paraensis (Miq.) Miq. & MORACEAE & 1 & 1 & 0 & 0 & 0 & 0 & 0 \\
\hline 328 & Ficus pertusa L. f. & MORACEAE & 0 & 0 & 0 & 1 & 1 & 4 & 0 \\
\hline 329 & Ficus trigona L. f. & MORACEAE & 0 & 2 & 0 & 0 & 0 & 0 & 2 \\
\hline 330 & Ficus trigonata $\mathrm{L}$. & MORACEAE & 1 & 0 & 0 & 0 & 0 & 0 & 0 \\
\hline 331 & Ficus ypsilophlebia Dugand & MORACEAE & 1 & 0 & 0 & 0 & 0 & 0 & 0 \\
\hline 332 & Ficus sp.1 & MORACEAE & 0 & 0 & 0 & 1 & 0 & 0 & 0 \\
\hline 333 & Ficus sp.2 & MORACEAE & 0 & 0 & 0 & 0 & 2 & 0 & 0 \\
\hline 334 & Helicostylis scabra (J.F. Macbr.) C.C. Berg & MORACEAE & 6 & 0 & 0 & 0 & 0 & 0 & 0 \\
\hline 335 & Helicostylis tomentosa (Poepp. \& Endl.) Rusby & MORACEAE & 3 & 0 & 0 & 0 & 0 & 0 & 0 \\
\hline 336 & Morus insignis Bureau & MORACEAE & 0 & 3 & 4 & 0 & 0 & 0 & 0 \\
\hline 337 & Perebea xanthochyma H. Karst. & MORACEAE & 1 & 0 & 0 & 0 & 1 & 0 & 0 \\
\hline 338 & Poulsenia armata (Miq.) Standl. & MORACEAE & 0 & 0 & 0 & 0 & 0 & 0 & 2 \\
\hline 339 & Pseudolmedia laevigata Trécul & MORACEAE & 1 & 0 & 0 & 0 & 0 & 0 & 4 \\
\hline 340 & Pseudolmedia laevis (Ruiz \& Pav.) J.F. Macbr. & MORACEAE & 4 & 0 & 0 & 15 & 14 & 0 & 0 \\
\hline 341 & $\begin{array}{l}\text { Pseudolmedia rigida (Klotzsch \& H. Karst.) } \\
\text { Cuatrec. }\end{array}$ & MORACEAE & 0 & 38 & 0 & 0 & 0 & 0 & 7 \\
\hline
\end{tabular}




\begin{tabular}{|c|c|c|c|c|c|c|c|c|c|}
\hline $\mathbf{N}^{0}$ & Especies & Familias & P-ST & P-PL & P-PR & P-GC & P-GL & P-MTL P & P-CPH \\
\hline 342 & Pseudolmedia sp. & MORACEAE & 0 & 0 & 0 & 0 & 0 & 0 & 2 \\
\hline 343 & Sorocea guillermiana Gaudich. & MORACEAE & 0 & 0 & 0 & 10 & 1 & 0 & 0 \\
\hline 344 & Trophis caucana (Pittier) C.C. Berg & MORACEAE & 0 & 0 & 0 & 25 & 13 & 25 & 0 \\
\hline 345 & Iryanthera juruensis Warb. & MYRISTICACEAE & 0 & 0 & 0 & 0 & 0 & 0 & 2 \\
\hline 346 & Otoba parvifolia (Markgr.) A.H. Gentry & MYRISTICACEAE & 0 & 0 & 0 & 2 & 35 & 2 & 4 \\
\hline 347 & Otoba sp. & MYRISTICACEAE & 0 & 0 & 0 & 0 & 0 & 0 & 4 \\
\hline 348 & Virola calophylla (Spruce) Warb. & MYRISTICACEAE & 0 & 0 & 0 & 2 & 11 & 0 & 1 \\
\hline 349 & Virola elongata (Benth.) Warb. & MYRISTICACEAE & 16 & 0 & 0 & 0 & 0 & 0 & 0 \\
\hline 350 & Virola sebifera Aubl. & MYRISTICACEAE & 18 & 0 & 0 & 0 & 0 & 0 & 1 \\
\hline 351 & Virola sp.1 & MYRISTICACEAE & 0 & 0 & 0 & 0 & 0 & 1 & 0 \\
\hline 352 & Virola surinamensis (Rol. ex Rottb.) Warb. & MYRISTICACEAE & 0 & 0 & 0 & 0 & 0 & 0 & 1 \\
\hline 353 & Virola theiodora (Spruce ex Benth.) Warb. aff. & MYRISTICACEAE & 8 & 0 & 0 & 0 & 0 & 0 & 0 \\
\hline 354 & Ardisia ambigua Mart. & MYRSINACEAE & 0 & 0 & 0 & 0 & 0 & 1 & 0 \\
\hline 355 & Cyabianthus sp. & MYRSINACEAE & 0 & 0 & 0 & 0 & 0 & 0 & 1 \\
\hline 356 & Cyabianthus sp.1 & MYRSINACEAE & 0 & 1 & 0 & 0 & 0 & 0 & 0 \\
\hline 357 & Cyabianthus sp.2 & MYRSINACEAE & 0 & 0 & 9 & 0 & 0 & 0 & 0 \\
\hline 358 & $\begin{array}{l}\text { Myrsine coriacea (Sw.) R. Br. ex Roem. \& } \\
\text { Schult. }\end{array}$ & MYRSINACEAE & 2 & 0 & 0 & 0 & 0 & 4 & 0 \\
\hline 359 & Myrsine guianensis (Aubl.) Kuntze & MYRSINACEAE & 0 & 6 & 2 & 0 & 0 & 0 & 0 \\
\hline 360 & Myrsine latifolia (Ruiz \& Pav.) Spreng. & MYRSINACEAE & 3 & 0 & 0 & 0 & 0 & 0 & 0 \\
\hline 361 & Myrsine oligophylla Zahlbr. & MYRSINACEAE & 0 & 4 & 0 & 0 & 0 & 0 & 0 \\
\hline 362 & Myrsine sp.1 & MYRSINACEAE & 0 & 0 & 1 & 5 & 0 & 0 & 0 \\
\hline 363 & Myrsine umbellata Mart. & MYRSINACEAE & 0 & 2 & 0 & 0 & 0 & 0 & 0 \\
\hline 364 & Stylogine cauliflora (Kunth) Mez & MYRSINACEAE & 0 & 4 & 0 & 0 & 0 & 0 & 0 \\
\hline 365 & Stylogine micrantha (Kunth) Mez & MYRSINACEAE & 0 & 0 & 0 & 1 & 0 & 0 & 0 \\
\hline 366 & Stylogine sp. & MYRSINACEAE & 0 & 0 & 0 & 0 & 0 & 0 & 1 \\
\hline 367 & Stylogine sp.1 & MYRSINACEAE & 0 & 0 & 1 & 0 & 0 & 0 & 0 \\
\hline 368 & Stylogine sp.2 & MYRSINACEAE & 0 & 0 & 1 & 0 & 0 & 0 & 0 \\
\hline 369 & Calyptranthes bipennis O. Berg & MYRTACEAE & 0 & 7 & 0 & 0 & 0 & 0 & 0 \\
\hline 370 & Calyptranthes speciosa Sagot & MYRTACEAE & 0 & 13 & 13 & 0 & 0 & 0 & 0 \\
\hline 371 & Calyptranthes sp. & MYRTACEAE & 1 & 0 & 0 & 0 & 0 & 0 & 0 \\
\hline 372 & Calyptranthes sp.1 & MYRTACEAE & 0 & 6 & 0 & 0 & 0 & 1 & 0 \\
\hline 373 & Calyptranthes sp.2 & MYRTACEAE & 0 & 0 & 13 & 0 & 0 & 0 & 0 \\
\hline 374 & Calyptranthes sp.4 & MYRTACEAE & 0 & 2 & 0 & 0 & 0 & 0 & 0 \\
\hline 375 & Eugenia uniflora L. & MYRTACEAE & 0 & 0 & 0 & 0 & 0 & 1 & 0 \\
\hline 376 & Eugenia sp. & MYRTACEAE & 0 & 0 & 0 & 0 & 0 & 0 & 5 \\
\hline
\end{tabular}




\begin{tabular}{|c|c|c|c|c|c|c|c|c|}
\hline $\mathbf{N}^{0}$ Especies & Familias & P-ST & P-PL & P-PR I & P-GC & P-GL & P-MTL $F$ & P-CPH \\
\hline 377 Eugenia sp.1 & MYRTACEAE & 0 & 0 & 0 & 0 & 0 & 1 & 0 \\
\hline 378 Eugenia sp.2 & MYRTACEAE & 0 & 0 & 0 & 3 & 0 & 0 & 0 \\
\hline 379 Eugenia $\mathrm{sp} .3$ & MYRTACEAE & 0 & 0 & 0 & 2 & 0 & 0 & 0 \\
\hline 380 Myrcianthes rhopaloides (Kunth) McVaugh & MYRTACEAE & 0 & 17 & 0 & 0 & 0 & 0 & 0 \\
\hline 381 Myrcianthes sp.1 & MYRTACEAE & 0 & 0 & 3 & 0 & 0 & 0 & 0 \\
\hline 382 Guapira myrtiflora (Standl.) Little & NYCTAGINACEAE & 1 & 0 & 0 & 0 & 0 & 0 & 0 \\
\hline 383 Neea sp.1 & NYCTAGINACEAE & 0 & 0 & 0 & 10 & 0 & 1 & 0 \\
\hline 384 Cespedesia spathulata (Ruiz \& Pav.) Planch. & OCHNACEAE & 0 & 0 & 0 & 0 & 0 & 2 & 0 \\
\hline 385 Heisteria acuminata (Bonpl.) Engl. & OLACACEAE & 0 & 0 & 0 & 6 & 0 & 0 & 0 \\
\hline 386 Heisteria ovata Benth. & OLACACEAE & 0 & 0 & 0 & 0 & 0 & 2 & 0 \\
\hline 387 Agonandra brasiliensis Miers ex Benth. & OPILIACEAE & 0 & 2 & 0 & 0 & 0 & 0 & 0 \\
\hline 388 Agonandra silvatica Ducke & OPILIACEAE & 0 & 0 & 0 & 2 & 0 & 0 & 0 \\
\hline 389 Bocconia integrifolia Bonpl. & PAPAVERACEAE & 0 & 0 & 0 & 0 & 0 & 0 & 2 \\
\hline 390 Piper calvescentinerve Trel. & PIPERACEAE & 0 & 3 & 3 & 0 & 0 & 0 & 0 \\
\hline 391 Piper heterophyllum Ruiz \& Pav. & PIPERACEAE & 0 & 19 & 0 & 0 & 0 & 0 & 0 \\
\hline 392 Piper reticulatum $\mathrm{L}$. & PIPERACEAE & 0 & 0 & 0 & 0 & 2 & 0 & 0 \\
\hline 393 Piper sp.1 & PIPERACEAE & 0 & 0 & 1 & 0 & 0 & 0 & 8 \\
\hline 394 Piper sp.2 & PIPERACEAE & 0 & 0 & 0 & 0 & 0 & 0 & 1 \\
\hline 395 Podocarpus oleifolius D. Don & PODOCARPACEAE & 0 & 1 & 0 & 0 & 0 & 0 & 0 \\
\hline 396 Prumnopitys harmsiana (Pilg.) de Laub. & PODOCARPACEAE & 0 & 2 & 0 & 0 & 0 & 0 & 0 \\
\hline 397 Coccoloba sp. & POLYGONACEAE & 0 & 0 & 0 & 0 & 0 & 0 & 3 \\
\hline 398 Triplaris americana L. & POLYGONACEAE & 0 & 0 & 0 & 0 & 2 & 9 & 0 \\
\hline 399 Triplaris setosa Rusby & POLYGONACEAE & 0 & 0 & 0 & 4 & 2 & 1 & 0 \\
\hline 400 Triplaris sp. & POLYGONACEAE & 0 & 0 & 0 & 0 & 0 & 0 & 4 \\
\hline 401 Rhamnidium elaeocarpum Reissek & RHAMNACEAE & 0 & 0 & 0 & 6 & 1 & 0 & 0 \\
\hline 402 Rhamnus sphaerosperma $\mathrm{Sw}$. & RHAMNACEAE & 0 & 0 & 0 & 12 & 0 & 1 & 0 \\
\hline 403 Prunus huantensis Pilg. & ROSACEAE & 0 & 0 & 0 & 1 & 0 & 0 & 0 \\
\hline 404 Prunus rigida Koehne & ROSACEAE & 0 & 0 & 0 & 0 & 0 & 0 & 2 \\
\hline 405 Prunus vana J.F. Macbr. & ROSACEAE & 0 & 6 & 2 & 7 & 0 & 0 & 0 \\
\hline 406 Bathysa peruviana K. Krause & RUBIACEAE & 0 & 0 & 0 & 0 & 0 & 2 & 0 \\
\hline 407 Bathysa sp.1 & RUBIACEAE & 0 & 0 & 1 & 1 & 0 & 0 & 0 \\
\hline $\begin{array}{l}\text { Calycophylum spruceanum (Benth.) Hook. f. ex } \\
\text { K. Schum. }\end{array}$ & RUBIACEAE & 0 & 0 & 0 & 0 & 1 & 0 & 0 \\
\hline 409 Capirona decorticans Spruce & RUBIACEAE & 10 & 0 & 0 & 0 & 0 & 0 & 0 \\
\hline 410 Chimarrhis hookerii K. Schum. & RUBIACEAE & 0 & 0 & 0 & 9 & 6 & 0 & 0 \\
\hline 411 Chimarrhis sp. 1 & RUBIACEAE & 0 & 0 & 0 & 0 & 1 & 0 & 0 \\
\hline
\end{tabular}




\begin{tabular}{|c|c|c|c|c|c|c|c|c|c|}
\hline $\mathbf{N}^{\mathbf{o}}$ & Especies & Familias & P-ST & P-PL & P-PR & P-GC & P-GL & P-MTL & P-CPH \\
\hline 412 & Condaminea corymbosa (Ruiz \& Pav.) DC. & RUBIACEAE & 0 & 0 & 0 & 0 & 0 & 1 & 0 \\
\hline 413 & Condaminea sp.1 & RUBIACEAE & 0 & 0 & 0 & 1 & 0 & 0 & 0 \\
\hline 414 & Cosmibuena grandiflora (Ruiz \& Pav.) Rusby & RUBIACEAE & 0 & 0 & 0 & 0 & 0 & 7 & 0 \\
\hline 415 & Elaeagia karstenii Standl. & RUBIACEAE & 0 & 0 & 4 & 0 & 0 & 0 & 0 \\
\hline 416 & Elaeagia sp.2 & RUBIACEAE & 0 & 0 & 4 & 0 & 0 & 0 & 0 \\
\hline 417 & Faramea multiflora A.Rich. & RUBIACEAE & 0 & 1 & 0 & 0 & 0 & 0 & 0 \\
\hline 418 & Guettarda hirsuta (Ruiz \& Pav.) Pers. & RUBIACEAE & 0 & 0 & 2 & 0 & 0 & 4 & 0 \\
\hline 419 & Guettarda sp.1 & RUBIACEAE & 0 & 0 & 1 & 0 & 0 & 0 & 0 \\
\hline 420 & $\begin{array}{l}\text { Ladenbergia oblongifolia (Humb. ex Mutis) L. } \\
\text { Andersson }\end{array}$ & RUBIACEAE & 2 & 0 & 0 & 4 & 0 & 56 & 1 \\
\hline 421 & Macbrideina peruviana Standl. BO & RUBIACEAE & 0 & 0 & 0 & 0 & 0 & 15 & 0 \\
\hline 422 & Macrocnemum roseum (Ruiz \& Pav.) Wedd. & RUBIACEAE & 0 & 0 & 0 & 0 & 0 & 2 & 0 \\
\hline 423 & Palicourea lasiantha K. Krause & RUBIACEAE & 1 & 0 & 0 & 0 & 0 & 0 & 0 \\
\hline 424 & Palicourea sp. & RUBIACEAE & 0 & 0 & 0 & 0 & 0 & 0 & 1 \\
\hline 425 & Palicourea sp.1 & RUBIACEAE & 0 & 0 & 0 & 0 & 0 & 1 & 0 \\
\hline 426 & Palicourea stipularis Benth. & RUBIACEAE & 0 & 19 & 0 & 0 & 0 & 0 & 0 \\
\hline 427 & Pentagonia sp. & RUBIACEAE & 0 & 0 & 0 & 0 & 0 & 0 & 1 \\
\hline 428 & Posoqueria sp.1 & RUBIACEAE & 0 & 0 & 3 & 0 & 0 & 0 & 0 \\
\hline 429 & Psychotria carthagenensis Jacq. & RUBIACEAE & 0 & 3 & 0 & 0 & 0 & 0 & 0 \\
\hline 430 & Psychotria sp. & RUBIACEAE & 0 & 0 & 0 & 0 & 0 & 0 & 3 \\
\hline 431 & Psychotria sp.1 & RUBIACEAE & 0 & 1 & 1 & 0 & 0 & 0 & 0 \\
\hline 432 & Psychotria sp.2 & RUBIACEAE & 0 & 1 & 0 & 0 & 0 & 0 & 0 \\
\hline 433 & Psychotria sp.3 & RUBIACEAE & 0 & 0 & 2 & 0 & 0 & 0 & 0 \\
\hline 434 & Randia armata (Sw.) DC. & RUBIACEAE & 0 & 0 & 0 & 1 & 1 & 0 & 0 \\
\hline 435 & Simira williansii (Standl.) Steyerm. & RUBIACEAE & 0 & 2 & 0 & 0 & 0 & 0 & 0 \\
\hline 436 & Warszewiczia coccinea (Vahl) Klotzsch & RUBIACEAE & 0 & 0 & 0 & 0 & 0 & 8 & 0 \\
\hline 437 & Neosprucea montana Cuatrec. & SALICACEAE & 0 & 1 & 0 & 0 & 0 & 0 & 0 \\
\hline 438 & Allophyllus floribundus (Poepp.) Radlk. & SAPINDACEAE & 0 & 5 & 0 & 0 & 0 & 0 & 0 \\
\hline 439 & Allophyllus pilosus (J.F.Macbr.) A.H.Gentry & SAPINDACEAE & 0 & 0 & 0 & 0 & 1 & 0 & 0 \\
\hline 440 & Allophyllus sp. & SAPINDACEAE & 0 & 0 & 0 & 0 & 0 & 0 & 4 \\
\hline 441 & Allophyllus sp.1 & SAPINDACEAE & 0 & 0 & 0 & 4 & 4 & 2 & 0 \\
\hline 442 & Allophyllus sp.2 & SAPINDACEAE & 0 & 0 & 1 & 4 & 0 & 0 & 0 \\
\hline 443 & Allophyllus sp.3 & SAPINDACEAE & 0 & 0 & 1 & 1 & 0 & 0 & 0 \\
\hline 444 & Allophyllus sp.4 & SAPINDACEAE & 2 & 0 & 0 & 0 & 0 & 0 & 0 \\
\hline 445 & Cupania cinerea Poepp. & SAPINDACEAE & 0 & 0 & 0 & 11 & 0 & 0 & 0 \\
\hline 446 & Cupania sp.1 & SAPINDACEAE & 0 & 4 & 4 & 0 & 0 & 0 & 0 \\
\hline
\end{tabular}




\begin{tabular}{|c|c|c|c|c|c|c|c|c|}
\hline $\mathbf{N}^{0}$ Especies & Familias & P-ST & P-PL & P-PR & P-GC & P-GL & P-MTI & P-CPH \\
\hline 447 Chrysophyllum argenteum Jacq. & SAPOTACEAE & 0 & 0 & 0 & 0 & 1 & 0 & 0 \\
\hline $\begin{array}{l}\text { Chrysophyllum venezuelanense (Pierre) } \\
\text { T.D.Penn. }\end{array}$ & SAPOTACEAE & 0 & 0 & 0 & 1 & 0 & 0 & 0 \\
\hline 449 Chrysophyllum sp. & SAPOTACEAE & 0 & 0 & 0 & 0 & 0 & 0 & 1 \\
\hline 450 Micropholis guyanensis (Bonpl.) Cogn. & SAPOTACEAE & 0 & 0 & 0 & 1 & 0 & 0 & 0 \\
\hline 451 Pouteria caimito (Ruiz \& Pav.) Radlk. & SAPOTACEAE & 0 & 0 & 0 & 6 & 3 & 0 & 0 \\
\hline 452 Pouteria lucuma (Ruiz \& Pav.) Kuntze & SAPOTACEAE & 0 & 1 & 0 & 0 & 0 & 0 & 0 \\
\hline 453 Pouteria sp. & SAPOTACEAE & 0 & 0 & 0 & 0 & 0 & 0 & 1 \\
\hline 454 Pouteria torta (Mart.) Radlk. & SAPOTACEAE & 0 & 0 & 0 & 1 & 0 & 0 & 0 \\
\hline 455 Cestrum auriculatum L'Hér. & SOLANACEAE & 0 & 0 & 0 & 0 & 0 & 0 & 12 \\
\hline 456 Cestrum racemosum Ruiz \& Pav. & SOLANACEAE & 0 & 1 & 0 & 0 & 0 & 0 & 0 \\
\hline 457 Cestrum sp. 2 & SOLANACEAE & 0 & 0 & 0 & 0 & 1 & 0 & 0 \\
\hline 458 Cyphomandra sp. & SOLANACEAE & 0 & 0 & 0 & 0 & 0 & 0 & 1 \\
\hline $\begin{array}{l}459 \text { Lycianthes cyathocalyx (Van Heurck \& Müll. } \\
\text { Arg.) Bitter }\end{array}$ & SOLANACEAE & 0 & 1 & 0 & 0 & 0 & 0 & 0 \\
\hline 460 Solanum americanum Mill. & SOLANACEAE & 0 & 1 & 0 & 0 & 0 & 0 & 0 \\
\hline $\begin{array}{l}\text { Solanum monadelphum Van Heurck \& Müll. } \\
\text { Arg. }\end{array}$ & SOLANACEAE & 0 & 0 & 1 & 0 & 0 & 0 & 0 \\
\hline 462 Solanum nemorense Dunal & SOLANACEAE & 0 & 0 & 1 & 0 & 0 & 0 & 0 \\
\hline 463 Solanum sp. 1 & SOLANACEAE & 0 & 2 & 0 & 0 & 1 & 0 & 5 \\
\hline 464 Sterculia frondosa Rich. & STERCULIACEAE & 0 & 0 & 0 & 8 & 4 & 1 & 0 \\
\hline 465 Sterculia sp.1 & STERCULIACEAE & 0 & 0 & 0 & 1 & 0 & 0 & 0 \\
\hline 466 Theobroma cacao L. & STERCULIACEAE & 0 & 0 & 0 & 6 & 3 & 0 & 0 \\
\hline 467 Huertea glandulosa Ruiz \& Pav. & STHAPHYLLIACEAE & 0 & 1 & 0 & 1 & 1 & 0 & 2 \\
\hline 468 Huertea sp.1 & STHAPHYLLIACEAE & 0 & 0 & 1 & 0 & 0 & 0 & 0 \\
\hline 469 Turpinia occidentalis (Sw.) G. Don & STHAPHYLLIACEAE & 0 & 0 & 3 & 0 & 0 & 0 & 2 \\
\hline 470 Styrax forma capparifolia & STYRACACEAE & 14 & 0 & 0 & 0 & 0 & 0 & 0 \\
\hline 471 Styrax guyanensis A.DC. & STYRACACEAE & 0 & 0 & 0 & 0 & 0 & 0 & 11 \\
\hline 472 Styrax pavonii A.DC. & STYRACACEAE & 0 & 1 & 3 & 0 & 0 & 0 & 3 \\
\hline 473 Styrax sp.1 & STYRACACEAE & 0 & 0 & 0 & 2 & 0 & 0 & 0 \\
\hline 474 Symplocus spruceana Gürke & SYMPLOCACEAE & 0 & 3 & 4 & 0 & 0 & 0 & 0 \\
\hline 475 Freziera sp. 1 & THEACEAE & 0 & 1 & 1 & 0 & 0 & 0 & 0 \\
\hline 476 Gordonia fruticosa (Schrad.) H. Keng & THEACEAE & 0 & 1 & 1 & 0 & 0 & 0 & 0 \\
\hline 477 Gordonia sp.1 & THEACEAE & 0 & 0 & 2 & 0 & 0 & 0 & 0 \\
\hline 478 Clavija sp. 1 & THEOPHRASTACEAE & 0 & 0 & 0 & 1 & 0 & 0 & 0 \\
\hline 479 Apeiba membranacea Spruce ex Benth. & TILIACEAE & 10 & 0 & 0 & 0 & 0 & 0 & 0 \\
\hline 480 Heliocarpus americanus L. & TILIACEAE & 0 & 4 & 0 & 2 & 2 & 3 & 10 \\
\hline 481 Celtis schipii Standl. & ULMACEAE & 0 & 0 & 0 & 6 & 11 & 1 & 0 \\
\hline
\end{tabular}




\begin{tabular}{|c|c|c|c|c|c|c|c|c|c|}
\hline $\mathbf{N}^{0}$ & Especies & Familias & P-ST & P-PL & P-PR & P-GC & P-GL & P-MTL & P-CPH \\
\hline 482 & Celtis sp. & ULMACEAE & 0 & 0 & 0 & 0 & 0 & 0 & 1 \\
\hline 483 & Trema micrantha (L.) Blume & ULMACEAE & 0 & 0 & 8 & 23 & 4 & 4 & 0 \\
\hline 484 & Myriocarpa sp. & URTICACEAE & 0 & 0 & 0 & 0 & 0 & 0 & 32 \\
\hline 485 & Myriocarpa stipitata Benth. & URTICACEAE & 0 & 0 & 0 & 11 & 0 & 0 & 3 \\
\hline 486 & Phenax sp.1 & URTICACEAE & 0 & 0 & 2 & 0 & 0 & 0 & 0 \\
\hline 487 & Urera baccifera (L.) Gaudich. & URTICACEAE & 0 & 1 & 0 & 0 & 0 & 0 & 0 \\
\hline 488 & Urera caracasana (Jacq.) Gaudich. ex Griseb. & URTICACEAE & 0 & 1 & 0 & 0 & 5 & 1 & 33 \\
\hline 489 & Urera sp. & URTICACEAE & 0 & 0 & 0 & 0 & 0 & 0 & 5 \\
\hline 490 & Urera sp.1 & URTICACEAE & 0 & 0 & 0 & 0 & 1 & 0 & 0 \\
\hline 491 & Aegiphila integrifolia (Jacq.) B.D. Jacks. & VERBENACEAE & 4 & 0 & 0 & 0 & 0 & 0 & 0 \\
\hline 492 & Aegiphila sp.1 & VERBENACEAE & 0 & 0 & 0 & 0 & 0 & 2 & 0 \\
\hline 493 & $\begin{array}{l}\text { Gloeospermum sphaerocarpum Triana \& } \\
\text { Planch. }\end{array}$ & VIOLACEAE & 0 & 0 & 0 & 0 & 0 & 1 & 0 \\
\hline 494 & Vochysia grandis Mart. & VOCHYSIACEAE & 0 & 0 & 0 & 1 & 0 & 0 & 0 \\
\hline 495 & Vochysia leguiana J.F. Macbr. & VOCHYSIACEAE & 0 & 0 & 0 & 0 & 0 & 7 & 0 \\
\hline 496 & Vochysia venulosa Warm. & VOCHYSIACEAE & 50 & 0 & 0 & 0 & 0 & 0 & 0 \\
\hline
\end{tabular}

